\title{
The anancomeric character of the pharmacophore 1,3,4- thiadiazoline framework in chiral spiro-cyclohexyl derivatives: effects on stereochemistry and spiro-junction lability. Thermodynamic aspects.
}

Sergio Menta, ${ }^{\dagger}$ Simone Carradori, ${ }^{\ddagger}$ Daniela Secci, ${ }^{\dagger}$ Cristina Faggi, ${ }^{\S}$ Luisa Mannina, ${ }^{\dagger}$ Roberto Cirilli*, ${ }^{*}$ and Marco Pierini. ${ }^{*} \dagger$

${ }^{\dagger}$ Dipartimento di Chimica e Tecnologie del Farmaco, Sapienza Università di Roma, P.le Aldo Moro 5, 00185 Rome, Italy

‡Department of Pharmacy, “G. D’Annunzio” University of Chieti-Pescara, Via dei Vestini 31, 66100 Chieti, Italy

SUniversità degli studi di Firenze, Dipartimento di Chimica, Via della Lastruccia 13, 50019

Sesto Fiorentino, Florence, Italy

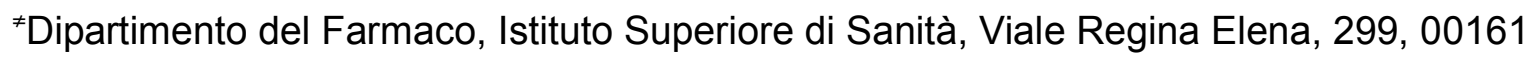

Rome, Italy

E-mails:

marco.pierini@uniroma1.it

roberto.cirilli@iss.it

Table of Contents



II. NMR assignments of the axial/equatorial alkyl arrangement in compounds $M-1 / 3$ and $L-1 / 3 \ldots \ldots \ldots \ldots \ldots . S-3$

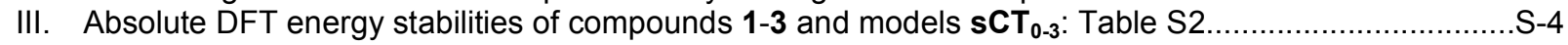

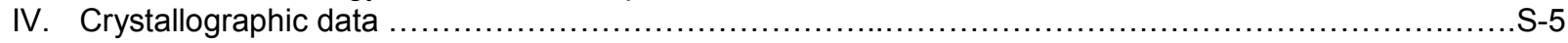

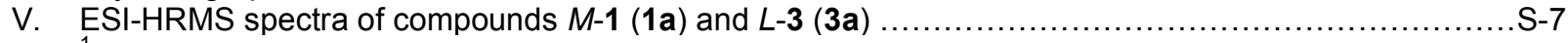

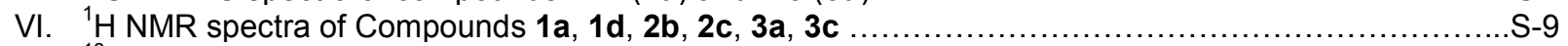

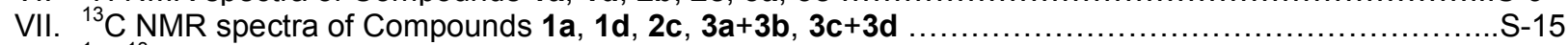

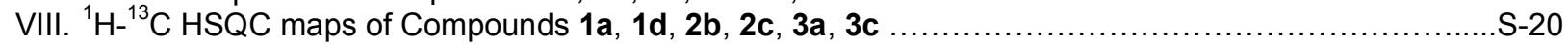




Scheme S1. General strategy for the absolute configuration assignment of the diastereomers $\mathbf{3 b}$ and $\mathbf{3 d}$. (a): synthesis of racemic 3-methylcyclohexanone thiosemicarbazone by a previously reported procedure (Chimenti, F.; Secci, D.; Bolasco, A.; Chimenti, P.; Granese, A.; Carradori, S.; Yanez, M.; Orallo, F.; Sanna, M. L.; Gallinella, B.; Cirilli, R. J. Med. Chem. 2010, 53, 6516-6520; (b): HPLC separation and absolute configuration determination of the enantiomers of 3-methylcyclohexanone thiosemicarbazone as previously reported (Carradori, S.; Cirilli, R.; Dei Cicchi, S.; Ferretti, R.; Menta, S.; Pierini, M.; Secci, D. J. Chromatogr. A 2012, $1269,168-177)$; (c) synthesis of the diastereomeric mixture $(5 R, 7 R)-3+(5 S, 7 R)-3$ starting only from enantiomer $R$ of the 3-methylcyclohexanone thiosemicarbazone; (d): evaluation of the elution order of the chromatographic peaks resulting from HPLC analysis of 3 spiked with the diastereomeric mixture $(5 R, 7 R)-3+$ (5S,7R)-3. Column: Chiralpak IC (250 mm x $4.6 \mathrm{~mm}$ d.i.); eluent: n-hexane-ethyl acetate-2-propanol 100:10:1 (v/v/v); detection: UV at $290 \mathrm{~nm}$; flow rate: $1.0 \mathrm{~mL} \mathrm{~min}^{-1}$; temperature: $25^{\circ} \mathrm{C}$.



$(R)$

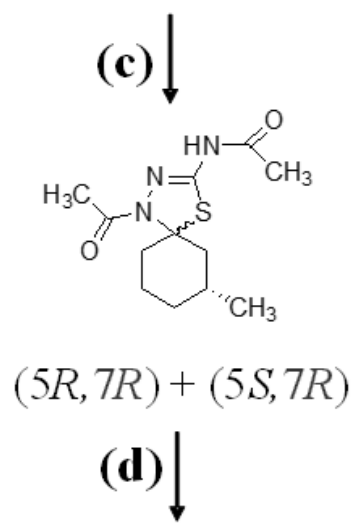

HPLC elution order: fourth $(5 R, 7 R)-\mathbf{3 d}$ and second $(5 S, 7 R)-\mathbf{3 b}$ eluting stereoisomers 


\section{NMR assignment of the conformational axial/equatorial arrangement assumed by the alkyl group linked}

to the cyclohexyl fragment in compounds $M-1 / 3$ and $L-1 / 3$.

All the requested assignments of axial/equatorial disposition assumed by the methyl or $t$-butyl group bonded to the cyclohexyl fragment have been based on suitable analysis of coupling constant values $\left(J_{a b}\right)$ relevant to protons sensible to the assumed geometries.

In the case of the more stable $M-2$ stereoisomer, $t$-butyl assumes an equatorial position. In fact, in this species, single proton $\mathrm{CH}-6$ is arranged in an axial disposition, as inferred from its $J_{a b}$ value of 12.5 Herz established through a typical axial-axial coupling with one of the protons $\mathrm{CH}-7$. Such an assignment was also confirmed by the $J_{a b}$ values measured for the axial $\mathrm{CH}-7$ proton, which are only compatible with arrangements of axial-axial type that it must assume with respect to protons $\mathrm{CH}-6$ and $\mathrm{CH}-8$ (12.7 Herz, $12.7 \mathrm{Herz})$, as well as with a geminal coupling established with the second proton bonded to carbon C-7 (12.7 Herz).

Also in the more stable $M-1$ and $M-3$ stereoisomers methyl group is found in an equatorial disposition, regardless of the position that it occupies on the cyclohexyl ring (i.e. on carbon C-6 or C-7). This is unambiguously suggested by the $J_{a b}$ values measured for the single proton in $\mathrm{CH}-6(M-1,13.1 \mathrm{~Hz})$ or $\mathrm{CH}-7(M-$ 3, $12.6 \mathrm{~Hz}$ ), only compatible with couplings of axial-axial type.

In turn, $J_{a b}$ values found for proton $\mathrm{CH}-7$ in compound $\mathrm{M}-1$ are only compatible with its axial-axial coupling with protons $\mathrm{CH}-6$ and $\mathrm{CH}-8(13.1 \mathrm{Herz}, 13.1 \mathrm{Herz})$, as well as with the geminal coupling with the second proton $\mathrm{CH}-$ $7(13.1 \mathrm{Herz}$ ), thus confirming the axial position assumed by proton $\mathrm{CH}-6$ in this species.

In the case of the less stable $L-1, L-2$ and $L-3$ stereoisomers, proton $J_{a b}$ values indicate that both the methyl and $t$-butyl groups preferentially assume an axial orientation on the cyclohexyl fragment. In compound $L-1$, the $J_{a b}$ values related to proton $\mathrm{CH}-7$ indicate axial-equatorial coupling with proton $\mathrm{CH}-6(4.3 \mathrm{~Hz})$, axial-axial coupling with proton $\mathrm{CH}-8(14.0 \mathrm{~Hz})$ and geminal coupling with proton $\mathrm{CH}-7(14.0 \mathrm{~Hz})$, so confirming the equatorial disposition possessed by the single proton $\mathrm{CH}-6$.

In compound $L-3$ the $J_{a b}$ values related to the axial proton $\mathrm{CH}-6$ point to an axial-equatorial coupling with proton $\mathrm{CH}-7(5.9 \mathrm{~Hz})$, as well as to a geminal coupling with second proton $\mathrm{CH}-6(14.0 \mathrm{~Hz})$, thus indirectly confirming the axial position assumed by the methyl group on C-7.

Finally, in the case of $L-2$, by $J_{a b}$ analysis, the unusual axial position assumed by the $t$-butyl group at the solid state was confirmed to be largely present also in solution. In fact, the couple of $J_{a b}$ that $\mathrm{CH}-6$ establishes with the two protons bonded on carbon C-7 (i.e. 8.7 and $3.7 \mathrm{~Hz}$ ) appears to be constituted by average values, arising from a mixture of two almost equally populated conformations of compound $L-2$, interconverting one to each other by fast flipping of the cyclohexyl ring. More in particular, according to the above picture, the $J_{a b} 8.7 \mathrm{~Hz}$ turns out to be compatible with an average value due to a weighted mix of equatorial-equatorial and axial-axial coupling constants, while the $J_{a b} 3.7 \mathrm{~Hz}$ with an average value coming from an axial-equatorial coupling that, after chair flipping, become equatorial-axial (i.e. without a change of typology of coupling).

This kind of interpretation results also coherent with what has been suggested by DFT calculations, which in the main text have been reported and commented within the subsection "Generation of Anancomeric Conformations". According to such findings, in fact, the conformational stereoisomers of L-2 that displays the proton $\mathrm{CH}-6$ either in equatorial or axial position have resulted characterized by Boltzmann populations of $60 \%$ and $40 \%$, respectively. 
Table S2. Absolute DFT energy stabilities of compounds 1-3 and models $\mathbf{S C T}_{0-3}$ computed at the $M 062 X / 6-31+G(d) / / B 3 L Y P / 6-31 G(d)$ level of theory.

\begin{tabular}{|c|c|c|c|}
\hline Compound & Configuration $^{a}$ & Conformation $^{b}$ & GS \\
\hline \multirow{4}{*}{1} & $\left(5 R^{*}, 6 S^{\star}\right)$ & alkyl $_{e q}, \mathrm{~S}_{a x}$ & -1181.30784 \\
\hline & $\left(5 R^{\star}, 6 S^{*}\right)$ & alkyl $_{a x}, \mathrm{~S}_{e q}$ & -1181.293355 \\
\hline & $\left(5 R^{*}, 6 R^{\star}\right)$ & alkyl $_{a x}, S_{a x}$ & -1181.301539 \\
\hline & $\left(5 R^{*}, 6 R^{\star}\right)$ & alkyl $_{e q}, S_{e q}$ & -1181.298624 \\
\hline \multirow{4}{*}{2} & $\left(5 R^{\star}, 6 R^{\star}\right)$ & alkyl $_{e q}, S_{a x}$ & -1299.181704 \\
\hline & $\left(5 R^{\star}, 6 R^{\star}\right)$ & alkyl $_{a x}, S_{e q}$ & -1299.160522 \\
\hline & $\left(5 R^{\star}, 6 S^{\star}\right)$ & alkyl $_{a x}, S_{a x}$ & -1299.170536 \\
\hline & $\left(5 R^{*}, 6 S^{*}\right)$ & alkyl $_{e q}, S_{e q}$ & -1299.170152 \\
\hline \multirow{4}{*}{3} & $\left(5 R^{*}, 7 R^{*}\right)$ & alkyl $_{e q}, S_{a x}$ & -1181.307748 \\
\hline & $\left(5 R^{*}, 7 R^{\star}\right)$ & alkyl $l_{a x}, S_{e q}$ & -1181.296056 \\
\hline & $\left(5 R^{*}, 7 S^{\star}\right)$ & alkyl $_{a x}, S_{a x}$ & -1181.302628 \\
\hline & $\left(5 R^{\star}, 7 S^{\star}\right)$ & alkyl $_{e q}, S_{e q}$ & -1181.299622 \\
\hline \multirow{2}{*}{$\mathbf{s C T}_{0}$} & - & $S_{a x}$ & -1142.011244 \\
\hline & - & $\mathrm{S}_{e q}$ & -1142.002881 \\
\hline \multirow{2}{*}{$\mathrm{sCT}_{1}$} & - & $S_{a x}$ & -934.0611608 \\
\hline & - & $\mathrm{S}_{\mathrm{eq}}$ & -934.0514464 \\
\hline \multirow{2}{*}{$\mathrm{sCT}_{2}$} & - & $S_{a x}$ & -781.4494479 \\
\hline & - & $\mathrm{S}_{e q}$ & -781.4483809 \\
\hline \multirow{2}{*}{$\mathrm{sCT}_{3}$} & - & $S_{a x}$ & -820.7387647 \\
\hline & - & $\mathrm{S}_{e q}$ & -820.7348422 \\
\hline
\end{tabular}

All calculations have been performed in chloroform, according to the SM8 solvation model.

${ }^{a}$ relative configuration of the structure; ${ }^{b}$ : axial or equatorial disposition assumed by the alkyl group and sulphur atom within each conformation. 
Crystallographic data

Compound 1d (Exp_616)

2(C12H19N3O2S $)+C 1.5 \mathrm{H} 300.5, \quad M=567.76$, Monoclinic, space group $C 2, a=26.012(1), \quad b=7.214(1)$, $c=20.211(1) \AA, \beta=128.649(1) V=2834.5(4) \AA 3, Z=4 \quad D c=1.273, \mu=1.980 \mathrm{~mm}-1, F(000)=1216$.

16221 reflections were collected with a $4.35<\theta<72.21$ range with a completeness to theta $98.5 \%$; 5311 were unique, the parameters were 352 and the final R index was 0.0380 for reflections having $I>2 \sigma l$, and 0.0406 for all data. Flack parameter is 0.01 (1) for the exact configuration.

Absolute configuration was established by anomalous-dispersion effects in diffraction measurement on the crystal.

Hydrogen atoms were all assigned in calculated positions, except hydrogens on N3a and N3b.

A colorless prismatic shaped crystal $(0.10 \times 0.08 \times 0.03)$ was used for collection.

In the asymmetric unit we have two independent molecules and a molecule of solvent (methanol) cocrystallized.

This methanol has the carbonyl group along the symmetry element

$\begin{array}{llll}\text { C1 } & 0.0000 & 0.95556 & 0.5000\end{array}$

$\begin{array}{llll}01 & 0.0000 & 0.78876 & 0.5000\end{array}$

so we have a fractional asymmetrical unit content.

In PARST analysis we find two strong hydrogen bonds. The first is intramolecular, the second is intermolecular between $\mathrm{N} 3 \mathrm{~b}-\mathrm{HNb}$ and $\mathrm{O} 1 \mathrm{a}$ in molecule reported for $(-\mathrm{x},+\mathrm{y},-\mathrm{z}+1)$ operation symmetry.

They have respectively the following parameters:

\begin{tabular}{|c|c|c|c|}
\hline N3A - -HNA & N3A $\quad \ldots$ O1B & HNA & N3A -HNA $\ldots$ O1B \\
\hline 0.894 & 2.726 & 1.849 & 166.47 \\
\hline N3B $-\mathrm{HNB}$ & N3B $\quad \ldots$ O1A & HNB $\ldots .01 \mathrm{~A}$ & N3B $\quad-H N B \quad \ldots$ O1A \\
\hline 0.804 & 2.755 & 1.966 & 166.88 \\
\hline
\end{tabular}

Compound 3c (Exp_617)

2(C12H19N3O2S), $M=538.72$, Monoclinic, space group $P$ 21, $a=8.058(1) b=14.090(1), c=12.214(1) \AA, \beta=$ 92.584(2) $V=1385.3(2) \AA 3, Z=2, \quad D c=1.291, \mu=2.076 \mathrm{~mm}-1, F(000)=576$.

7997 reflections were collected with a $4.79<\theta<71.90$ range with a completeness to theta $96.7 \%$; 4411 were independent, the parameters were 333 and the final $R$ index was 0.0394 for reflections having $I>2 \sigma l$, and 0.0448 for all data. Flack parameter is $-0.01(2)$ for the exact configuration.

Absolute configuration was established by anomalous-dispersion effects in diffraction measurement on the crystal.

Hydrogen atoms were all assigned in calculated positions, except hydrogens on N3a and N3b.

A colourless cubic shaped crystal $(0.08 \times 0.07 \times 0.04)$ was used for collection; also in this case in the asymmetric unit we have two independent molecules.

In PARST analysis we find a strong intermolecular hydrogen bond between $\mathrm{N} 3 \mathrm{~b}-\mathrm{HNb}$ and $\mathrm{O} 1 \mathrm{a}$ in molecule reported for $(-x+2,+y+1 / 2,-z+2)$ operation symmetry. It has the following parameters:

\begin{tabular}{|l|l|ll|ll|}
\hline N3B $-\mathrm{HNB}$ & $\mathrm{N} 3 \mathrm{~B} \ldots \mathrm{O} 1 \mathrm{~A}$ & $\mathrm{HNB} \quad \ldots$ O1A & N3B $-\mathrm{HNB} \quad \ldots$...1A \\
\hline 0.878 & 2.900 & 2.026 & 174.24 & \\
\hline
\end{tabular}

The previous two set of data were collected at $150^{\circ} \mathrm{K}$.

Compound 2b (Exp_714)

C15H25N3O2S, M=311.44, Orthorhombic, space group P 2121 21, a=9.151(1), b=10.670(1), c=17.149(1)A,, $V=1674.4(3) \AA 3, Z=4 \quad D c=1.235, \mu=1.783 \mathrm{~mm}-1, F(000)=672$.

6525 reflections were collected with a $4.88<\theta<71.23$ range with a completeness to theta $97.2 \%$; 3087 were independent, parameters were 194 and final $R$ index is 0.0341 considering reflections having $\mathrm{I}>2 \sigma \mathrm{l}$, and 0.0434 for all data. Flack parameter is $-0.01(2)$ for the exact configuration.

Absolute configuration was established by anomalous-dispersion effects in diffraction measurement on the crystal. 
Hydrogen atoms were all assigned in calculated positions, except hydrogens on N3.

A colorless prismatic shaped crystal $(0.08 \times 0.06 \times 0.03)$ was used for collection.

In conformational analysis, using PARST, we find a strong intermolecular hydrogen bond between N3-HN3 and $01 a$ in molecule reported for $(x-1 / 2,+y-1 / 2,+z)$ operation symmetry.

Parameters are reported below:

\begin{tabular}{|l|l|l|l|}
\hline N3 -HN3 & N3 ... 1 & HN3 ... 1 & N3 $-\mathrm{HN} 3 \ldots$. 1 \\
\hline 0.818 & 2.752 & 1.940 & 172.4 \\
\hline
\end{tabular}

Compound 2c (Exp_713)

C15H25N3O2S, M=311.44, Orthorhombic, space group P 2121 21, a=9.602(1), $b=11.794(1), c=15.017(1) \AA$, $V=1700.6(3) \AA 3, Z=4 \quad D c=1.216, \mu=1.755 \mathrm{~mm}-1, F(000)=672$.

9397 reflections were collected with a $4.77<\theta<72.15$ range with a completeness to theta $97.5 \%$; 3220 were independent, parameters were 195 and final $R$ index is 0.0387 considering reflections having $I>2 \sigma \mathrm{l}$, and 0.0537 for all data. Flack parameter is $-0.03(2)$ for the exact configuration.

Absolute configuration was established by anomalous-dispersion effects in diffraction measurement on the crystal.

Hydrogen atoms were all assigned in calculated positions, except hydrogens on N3.

Also in this case, in conformational analysis using PARST, we note a strong intermolecular hydrogen bond between N3-HN3 and O1a in molecule reported for $(x+1 / 2,-y+1 / 2,-z+1)$ operation symmetry.

Parameters are reported below:

\begin{tabular}{|l|l|l|l|}
\hline N3 $-\mathrm{HN} 3$ & $\mathrm{~N} 3 \ldots \mathrm{O} 1$ & $\mathrm{HN} 3 \ldots \mathrm{O} 1$ & $\mathrm{~N} 3 \quad \mathrm{HN} 3 \quad \ldots \mathrm{O} 1$ \\
\hline 0.791 & 2.970 & 2.203 & 163.4 \\
\hline
\end{tabular}

These second two set of data were collected at room temperature. 


\section{ESI-HRMS experiments.}

\section{Compound 1a}

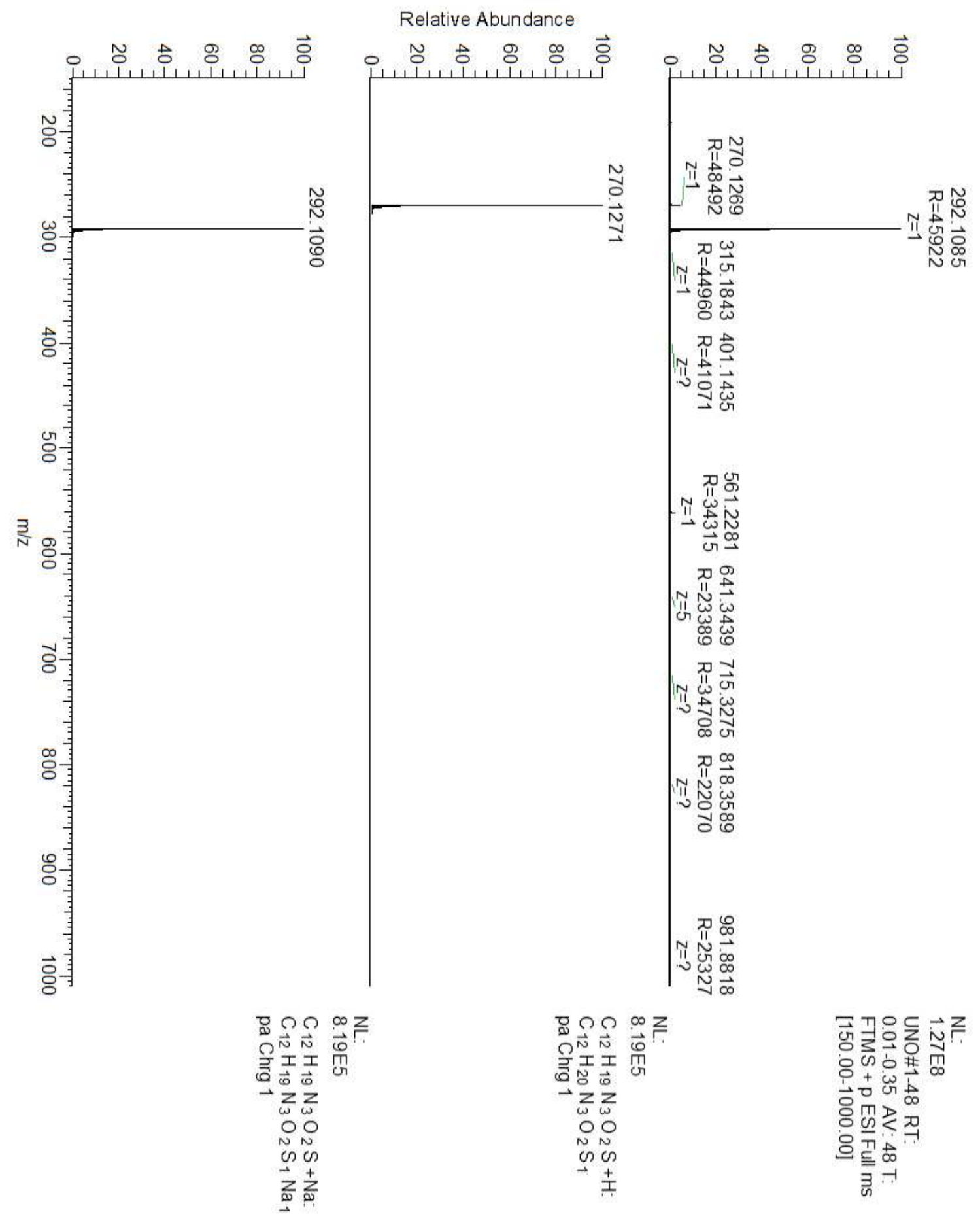


Combound $3 a$

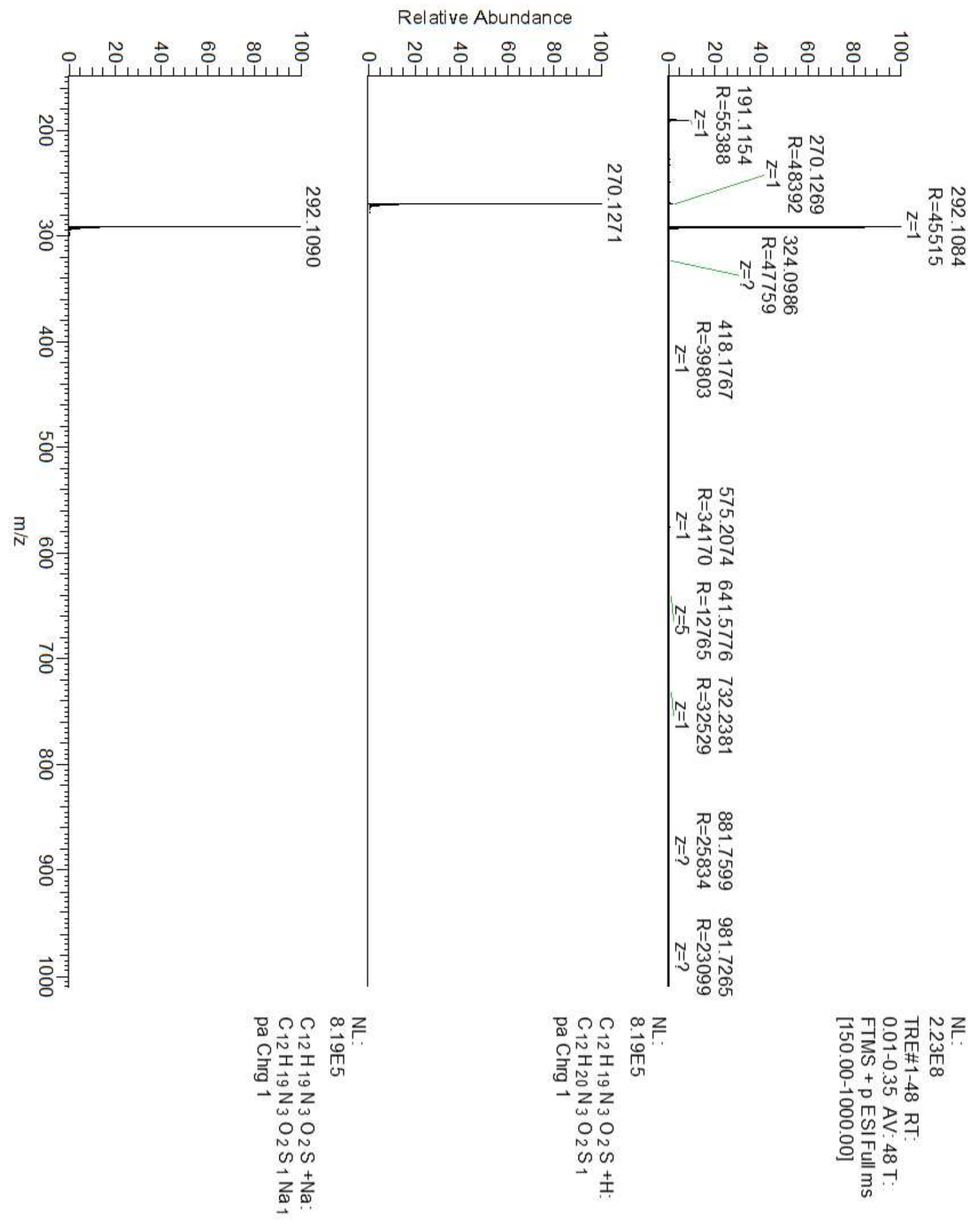




\section{${ }^{1} \mathrm{H}$ NMR spectra of Compounds $1 \mathbf{a}, \mathbf{1 d}, \mathbf{2 b}, \mathbf{2 c}, \mathbf{3 a}, \mathbf{3 c}$ Compound 1a}

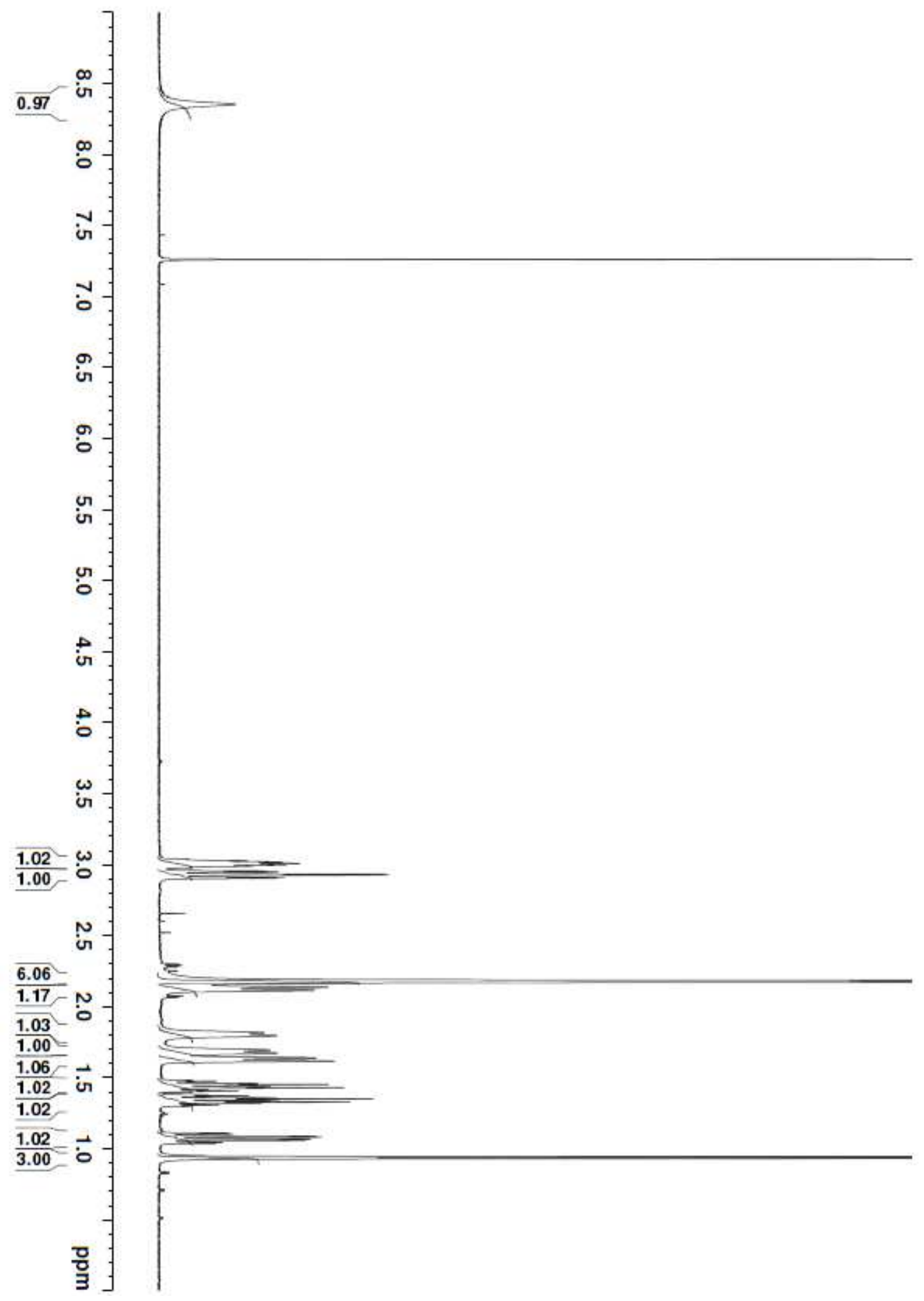


Compound 1d

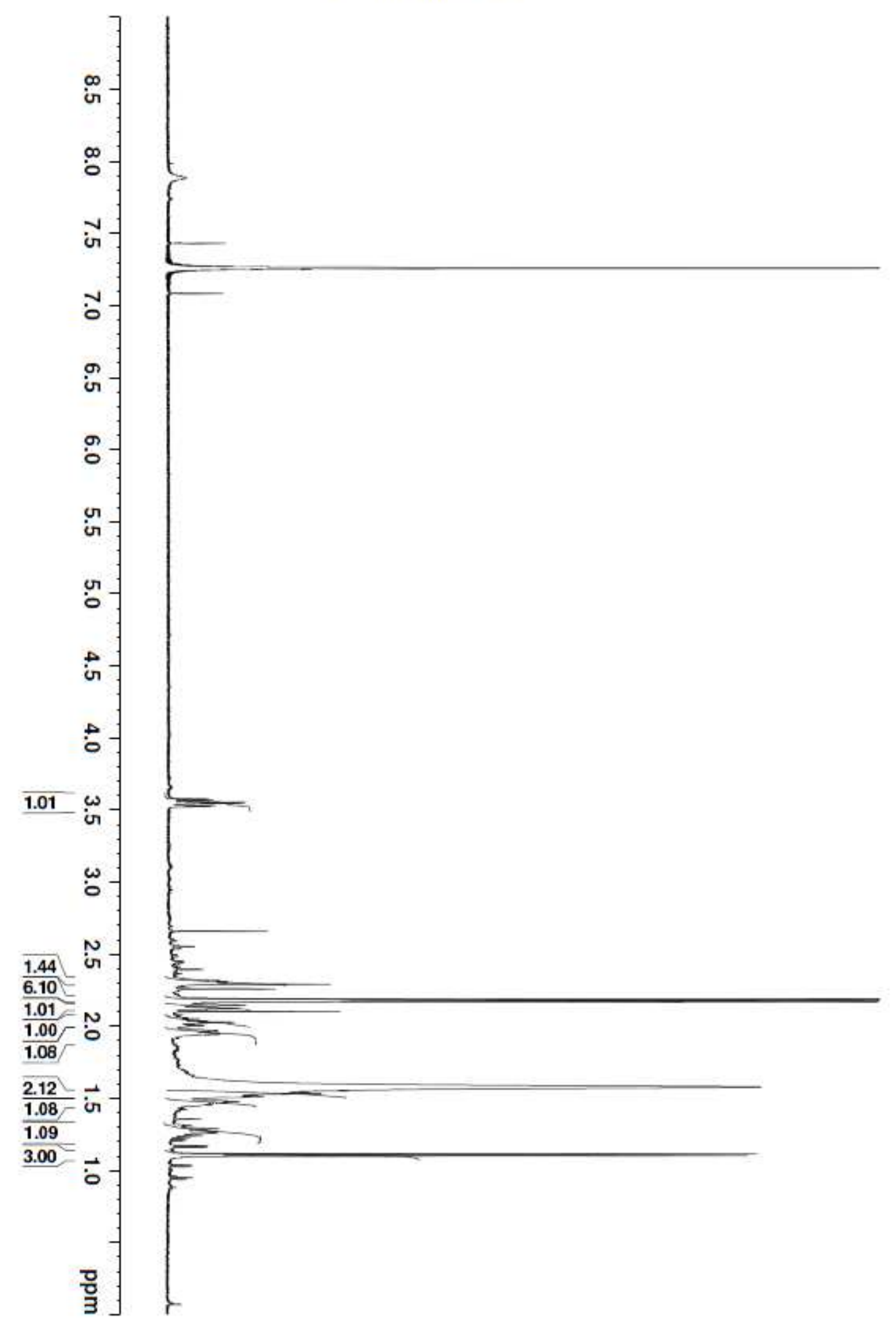


Compound 2b

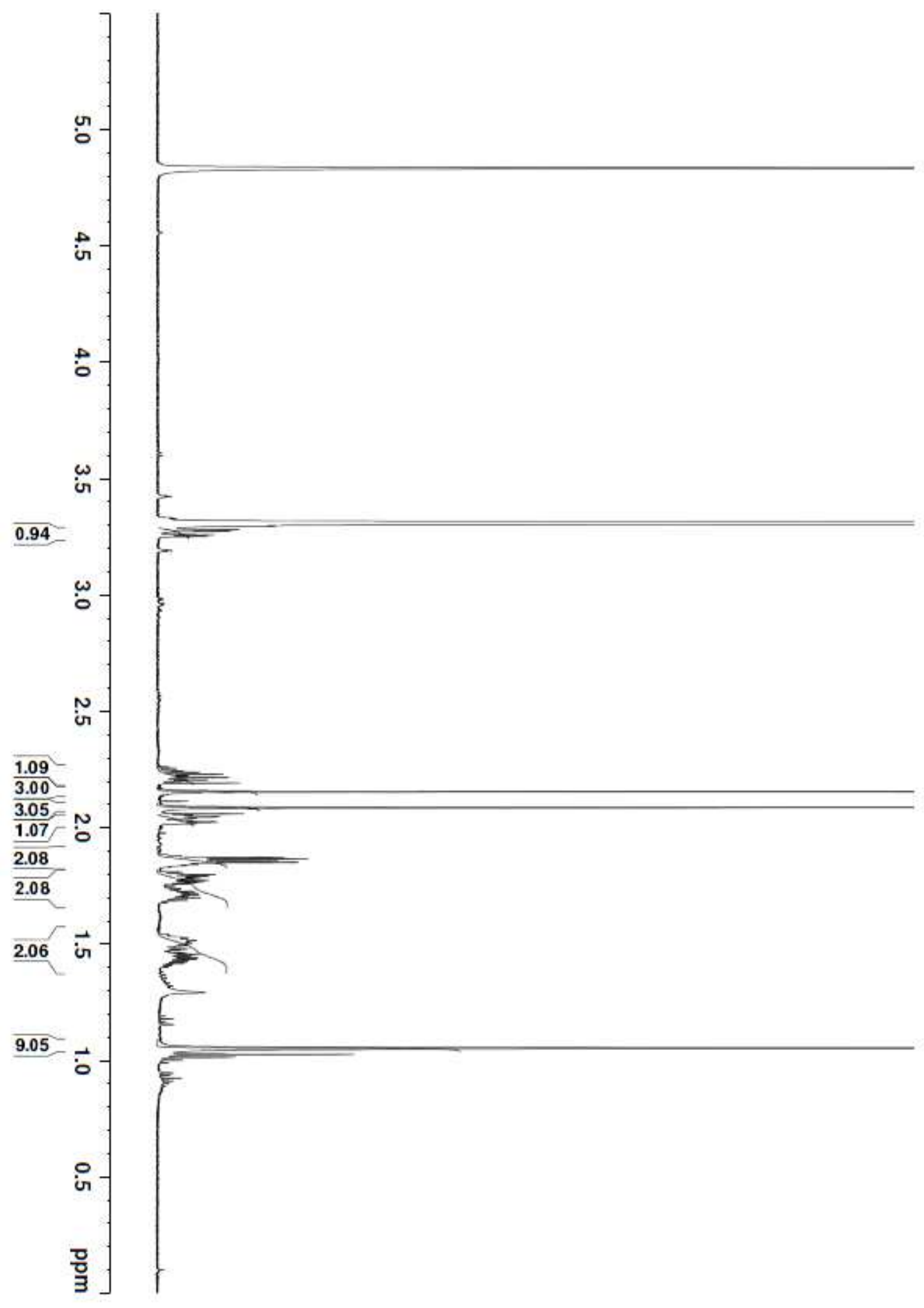


Compound 2c

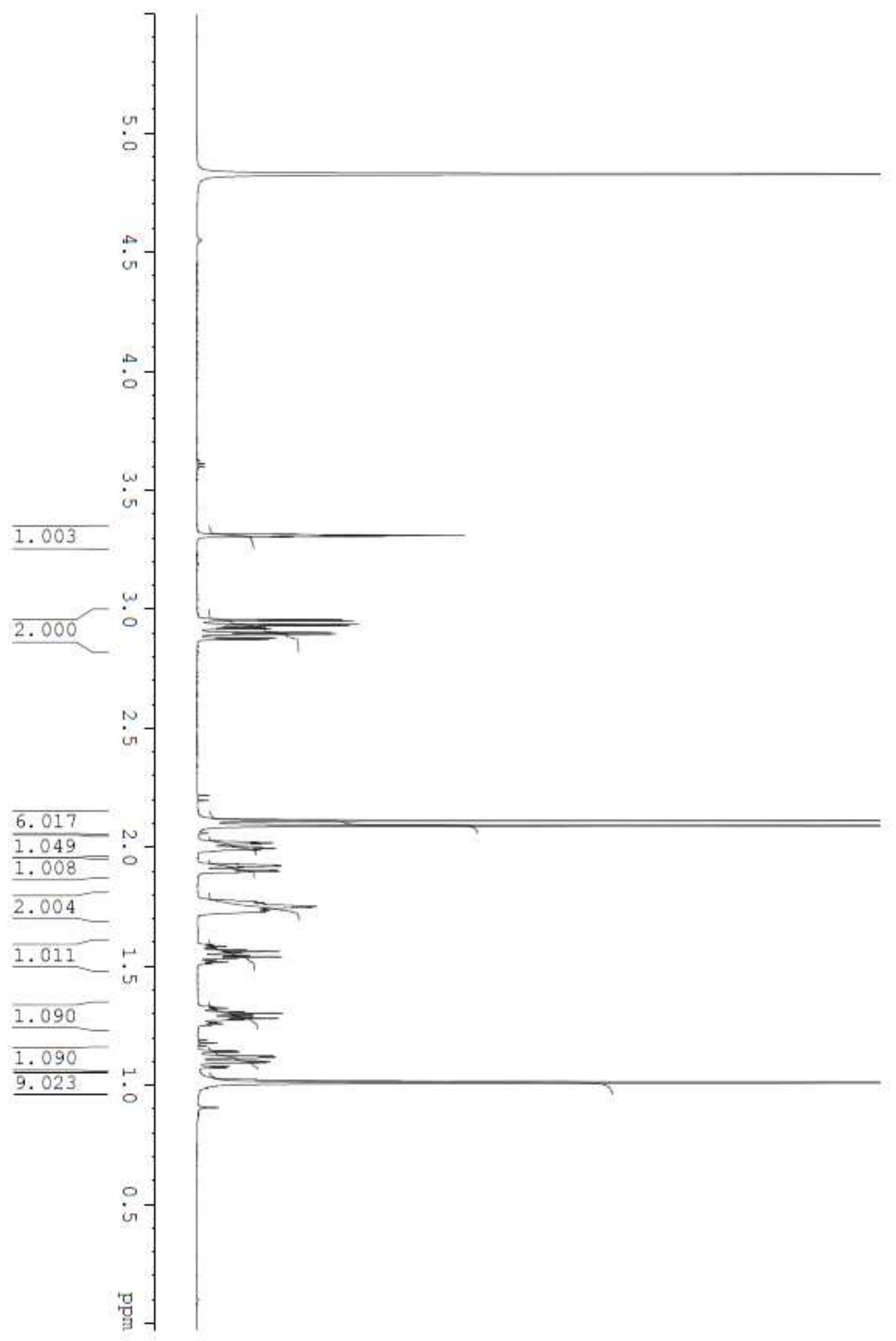


Compound 3a

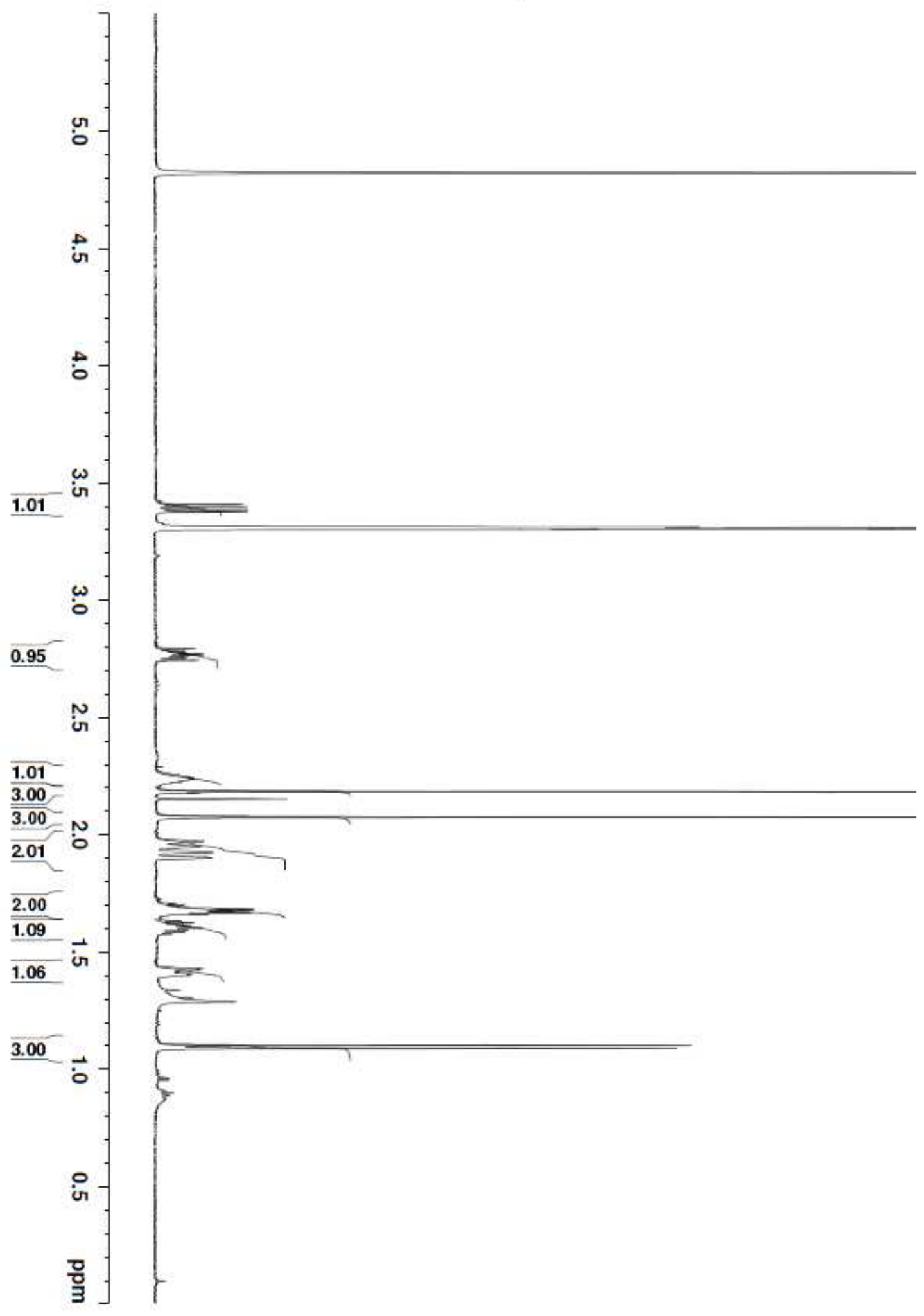


Compound 3c

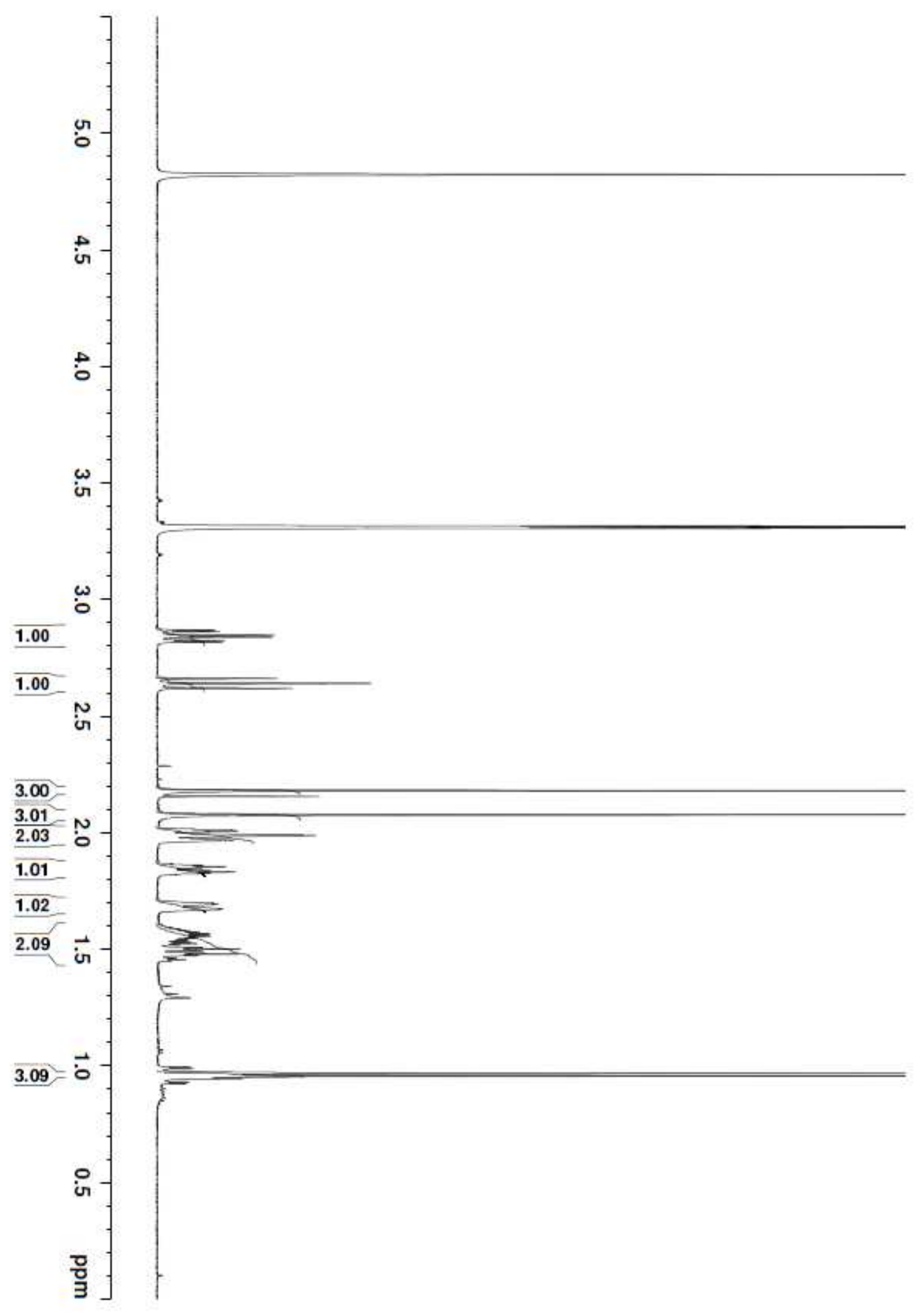


${ }^{13} \mathrm{C}$ NMR spectra of Compounds $1 \mathbf{a}, \mathbf{1 d}, \mathbf{2 c}, \mathbf{3 a}+\mathbf{3 b}, \mathbf{3 c}+\mathbf{3 d}$

Compound 1a

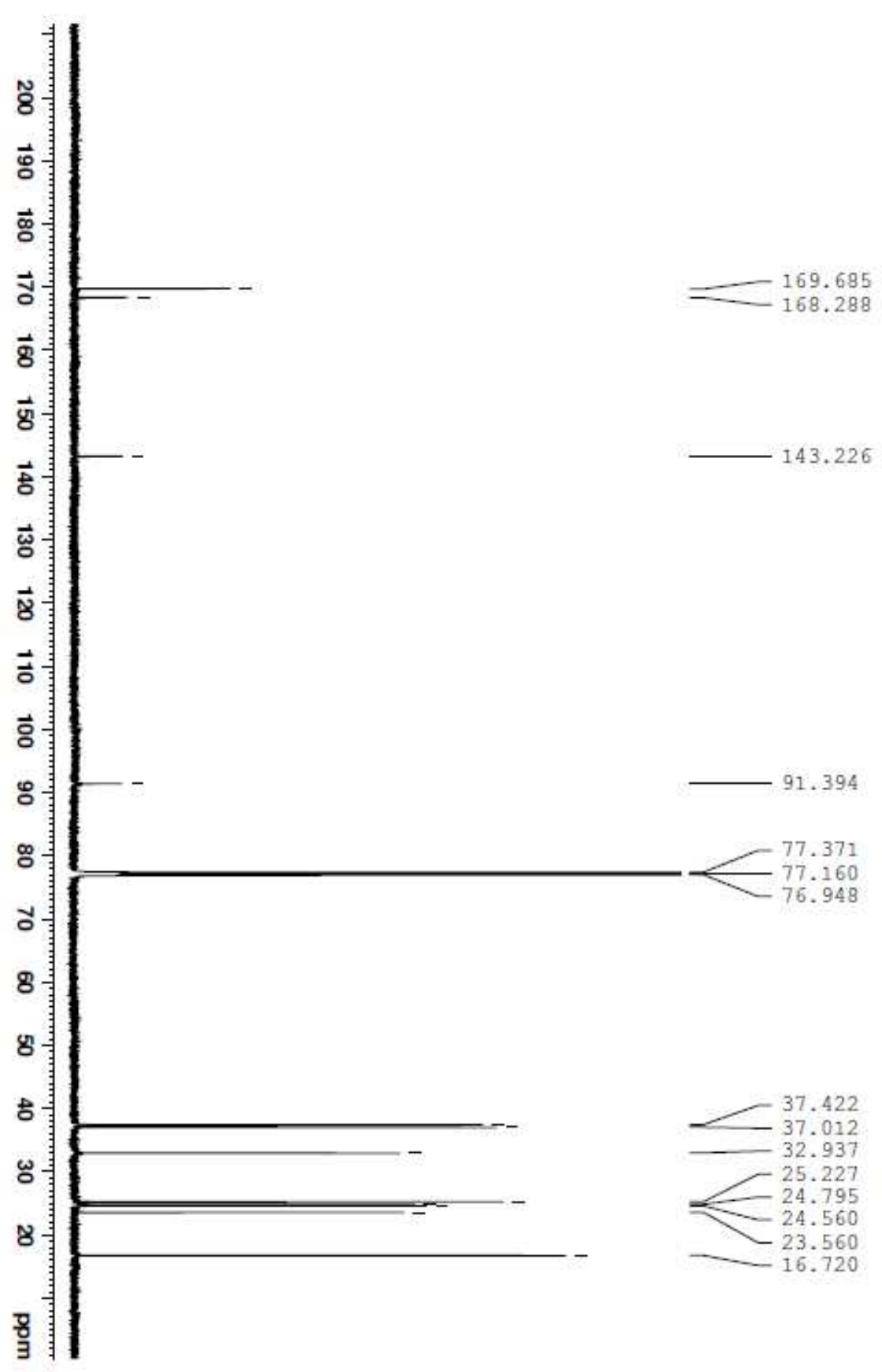


Compound 1d

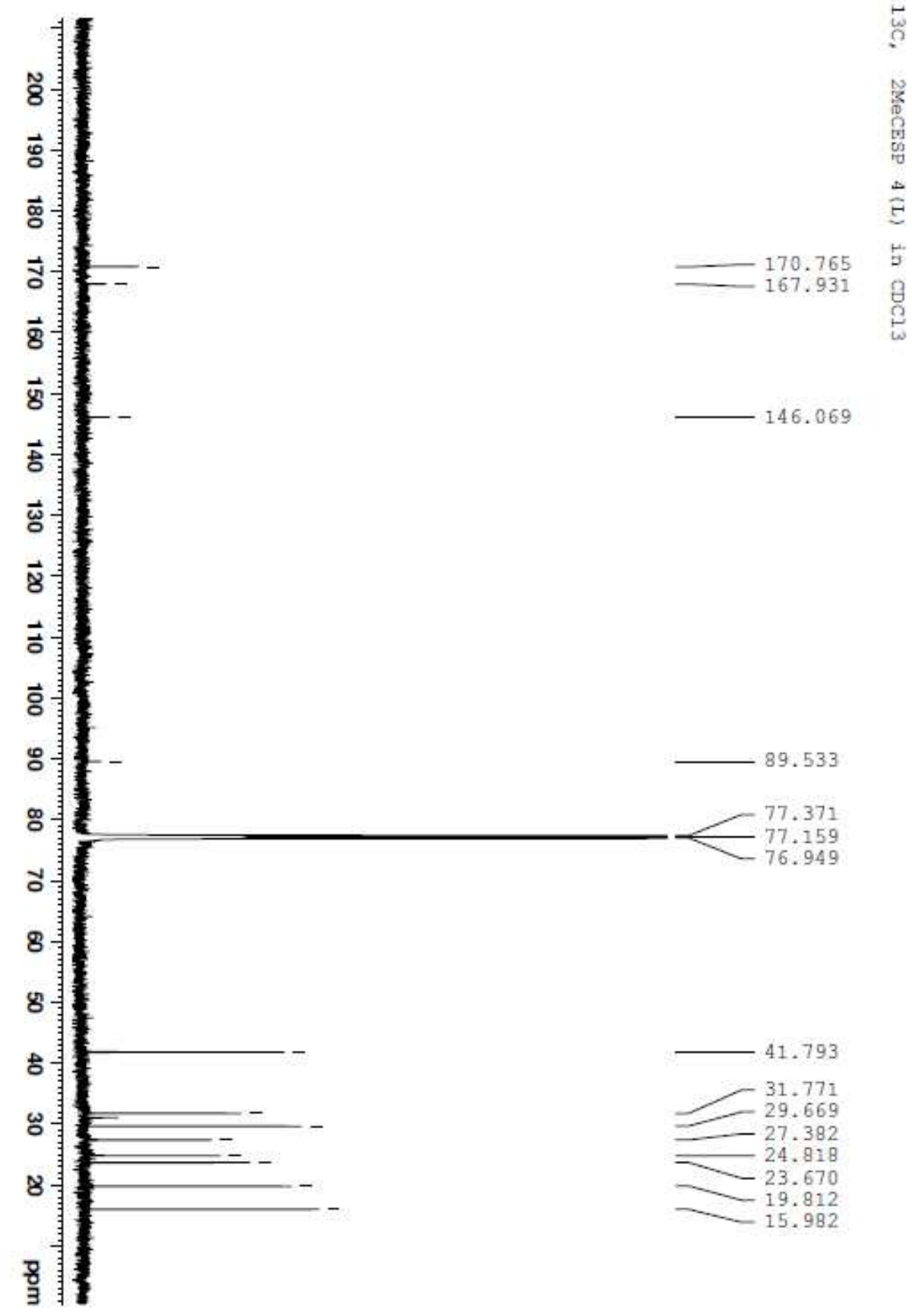


Compound 2c

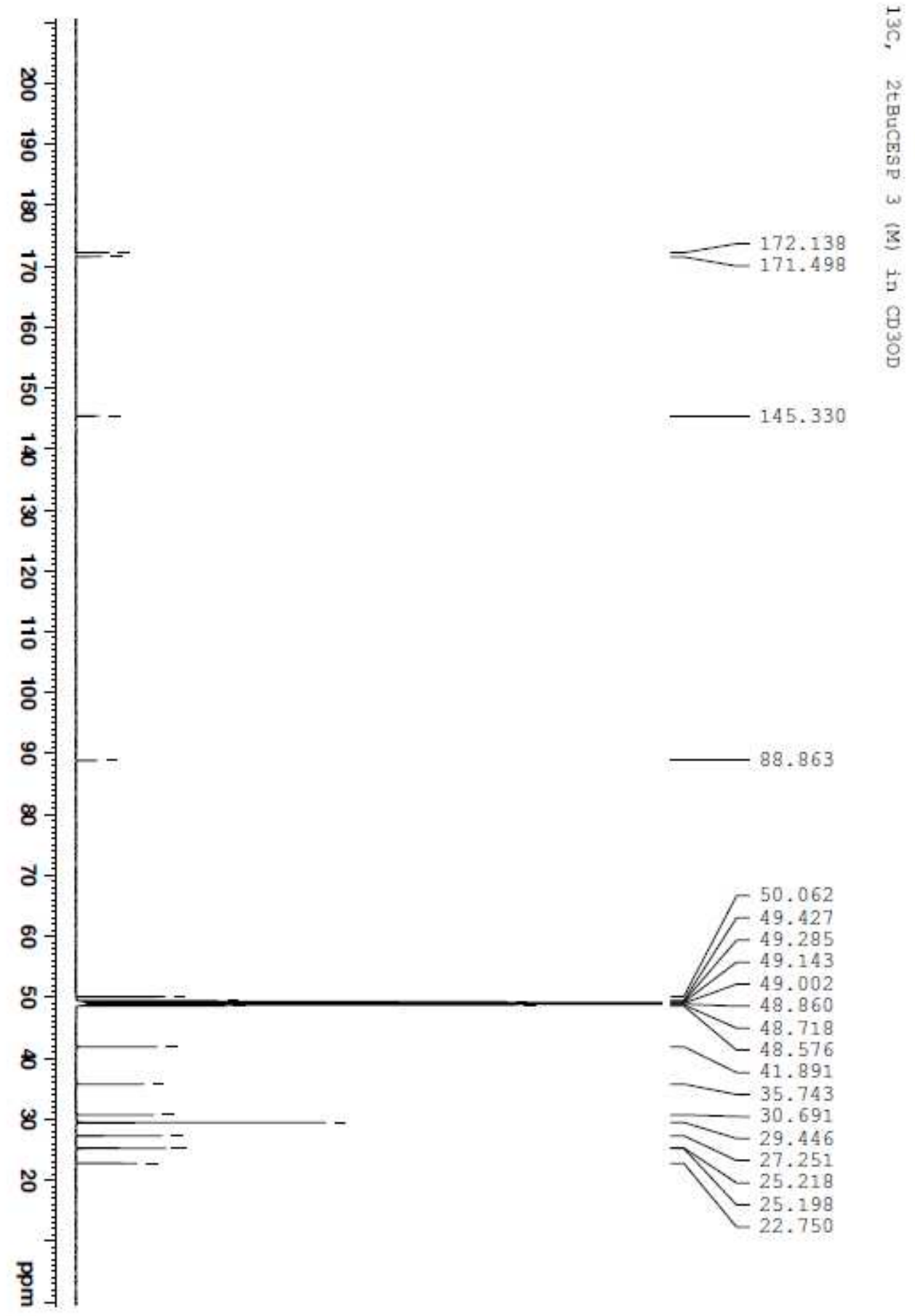


Compounds $3 a+3 b$

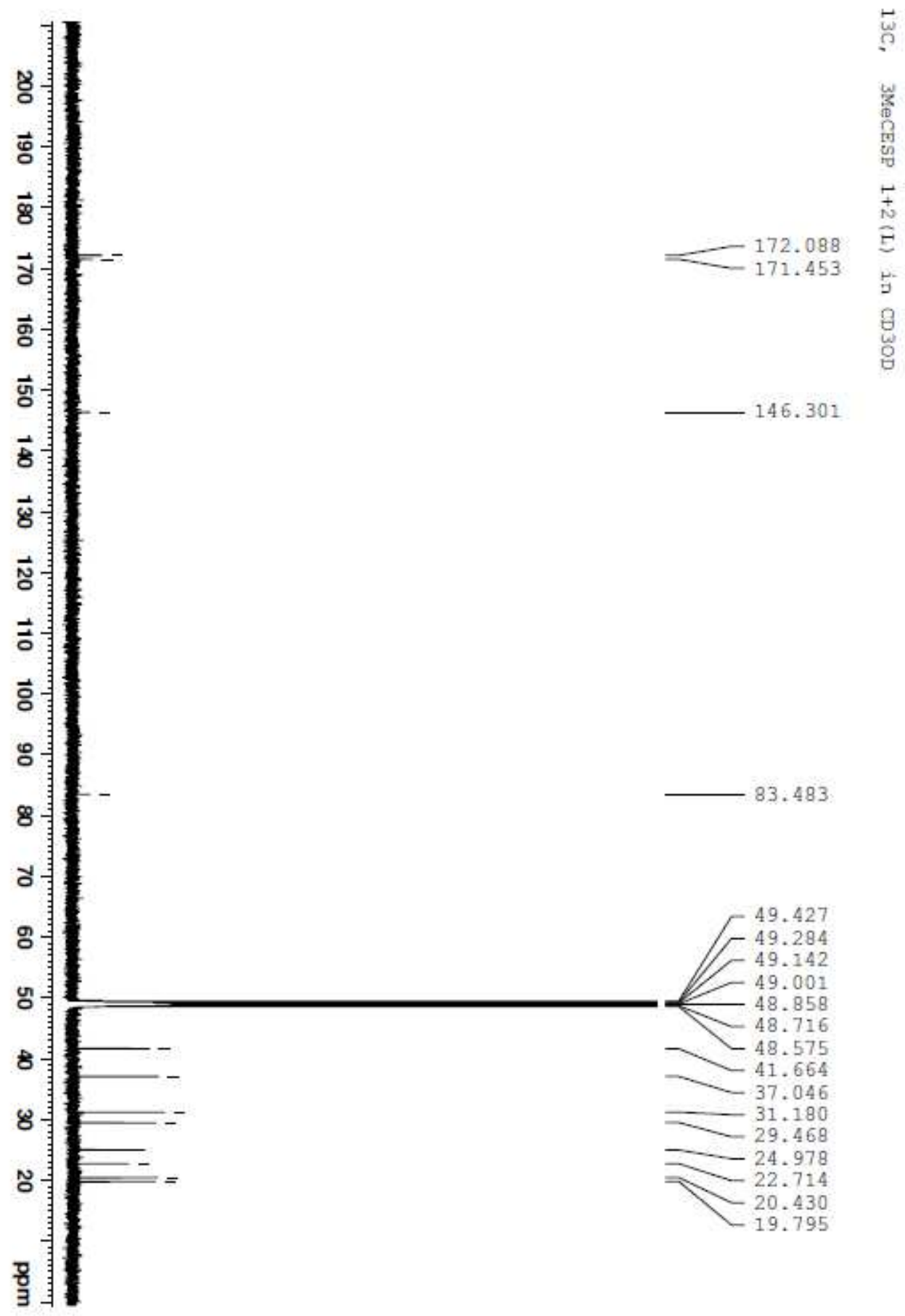


Compounds $3 c+3 d$

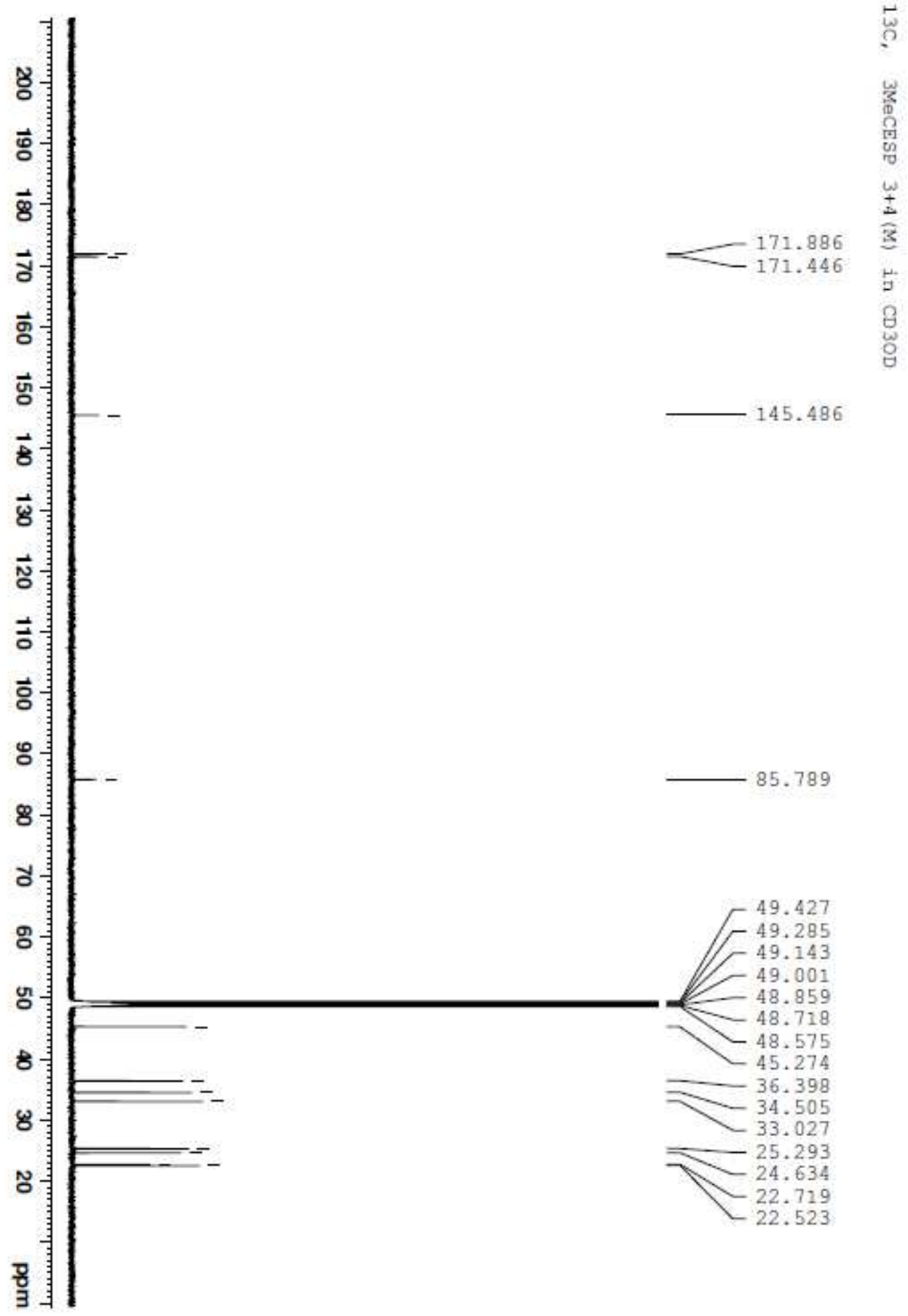


${ }^{1} \mathrm{H}-{ }^{13} \mathrm{C}$ HSQC maps of Compounds 1a, 1d, 2b, 2c, 3a, 3c

Combound 1a

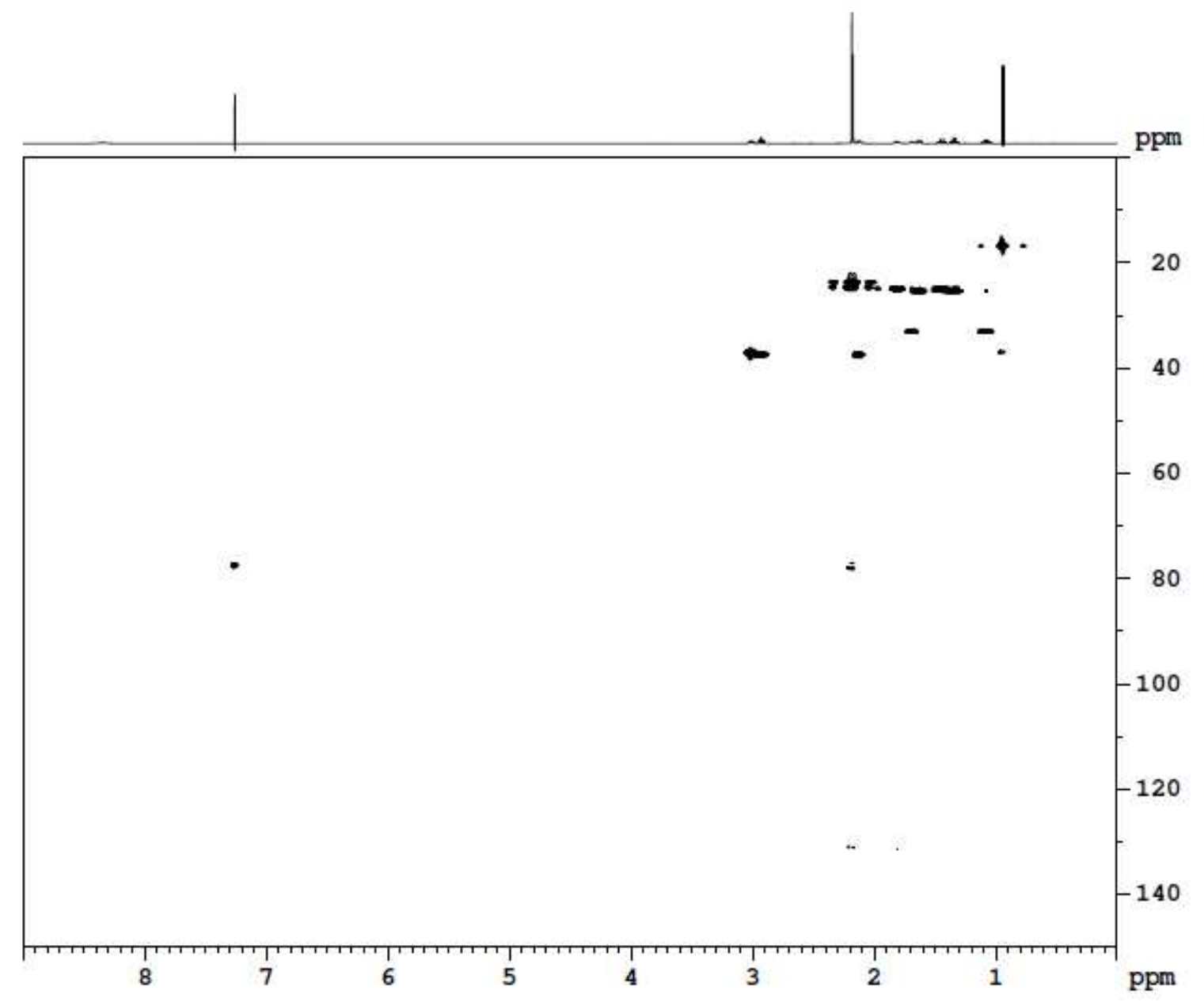




\section{Compound 1d}

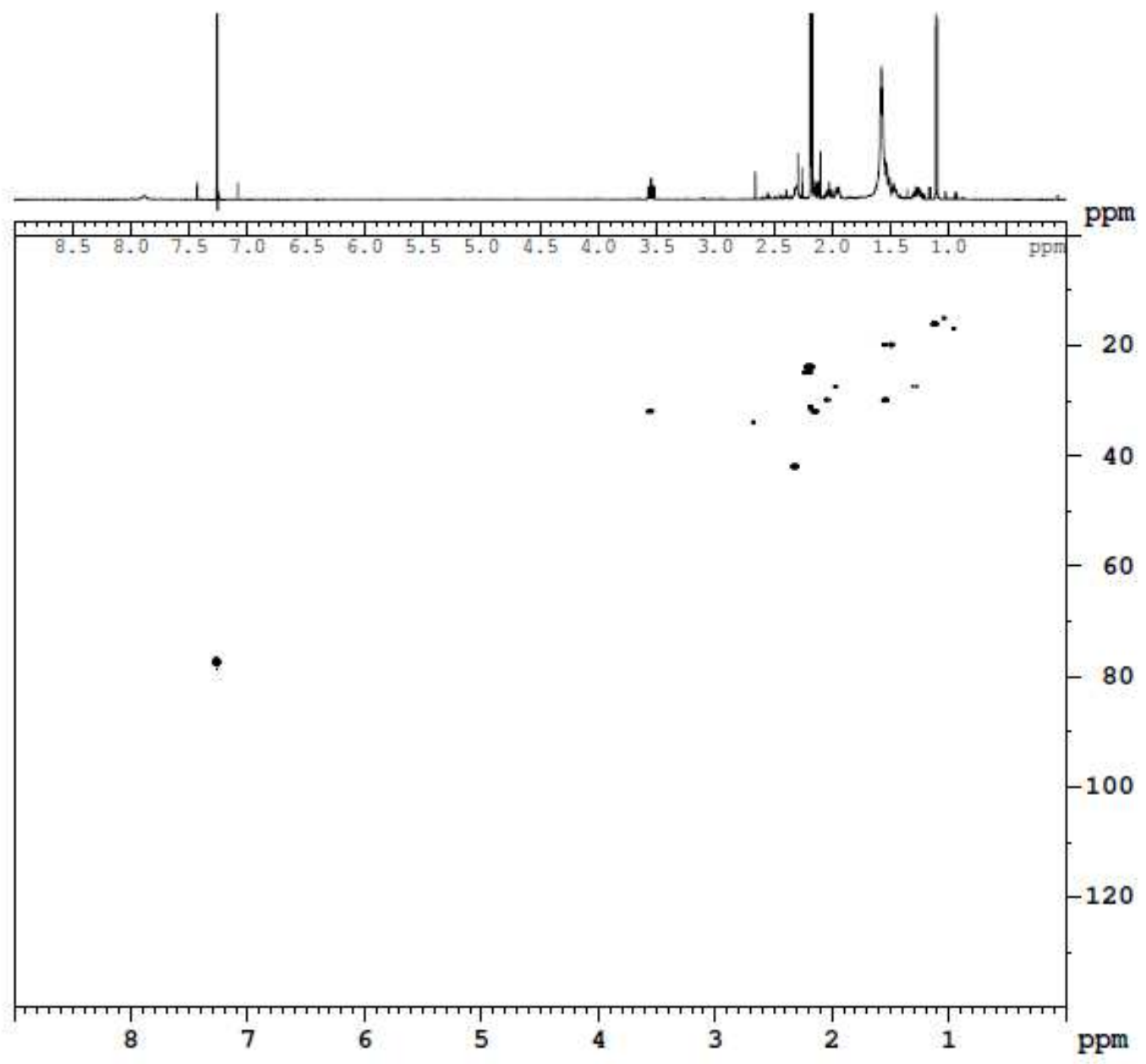


Compound $\mathbf{2 b}$

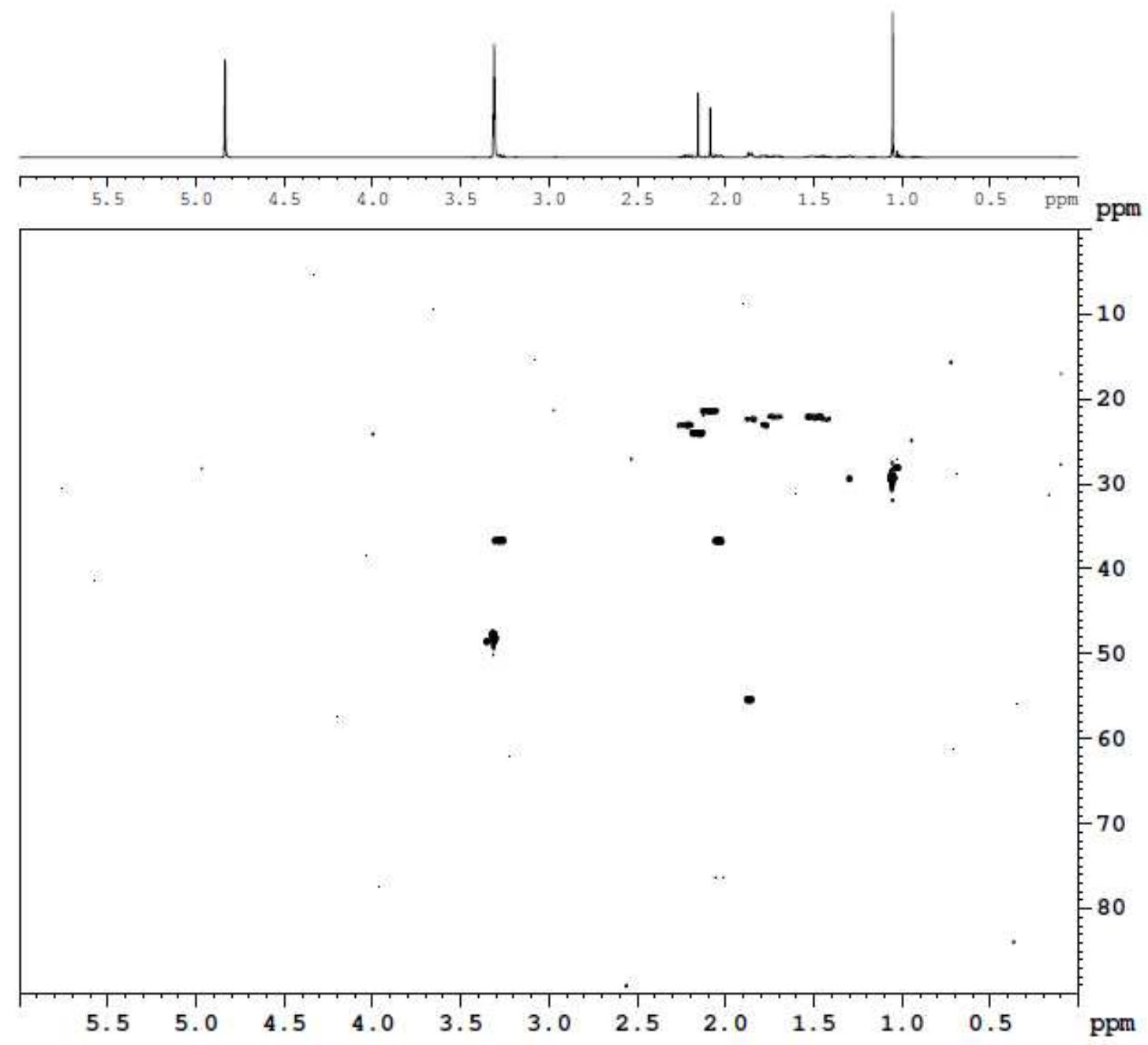


Combound 2c

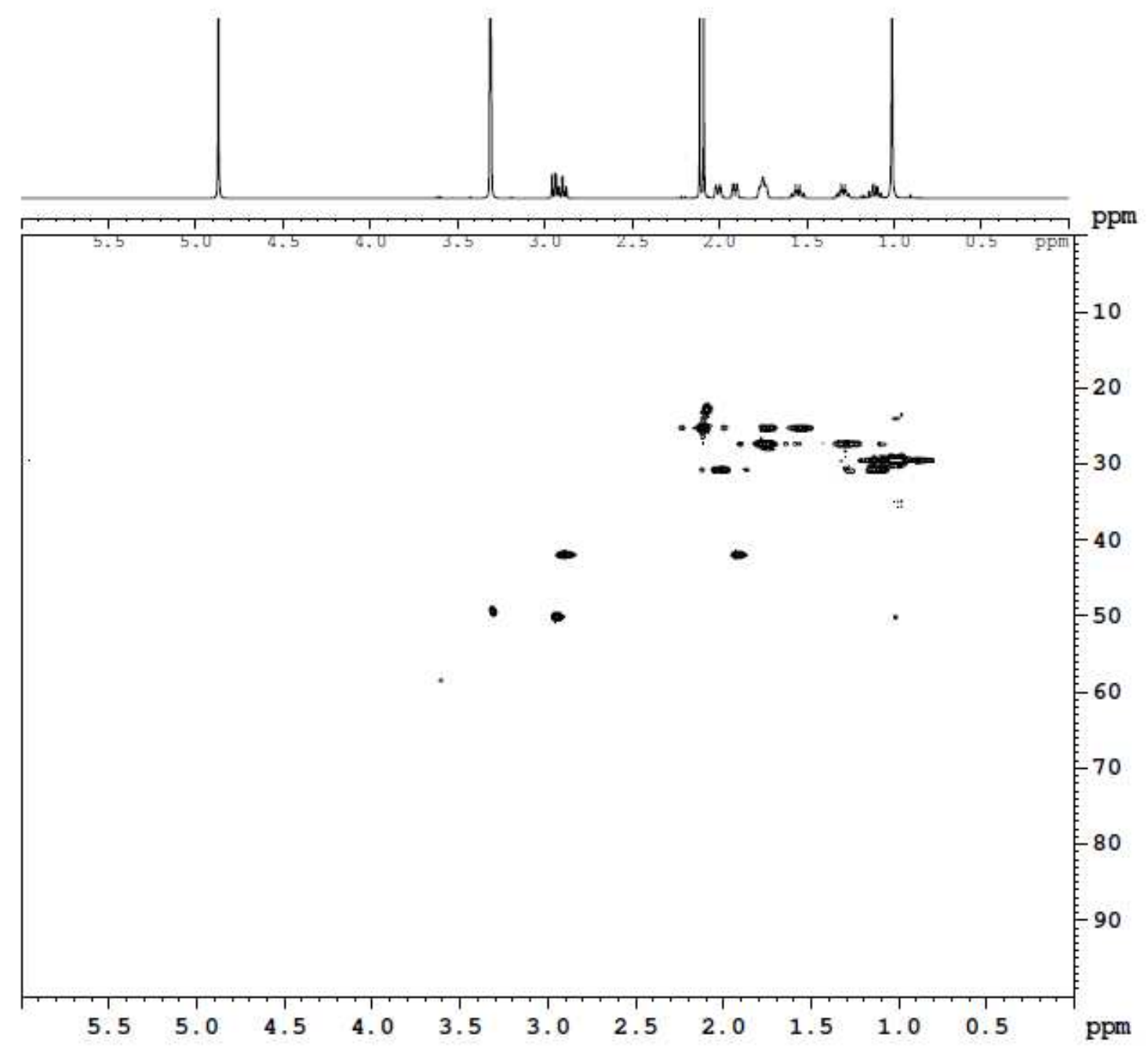


Compound $\mathbf{3 a}$

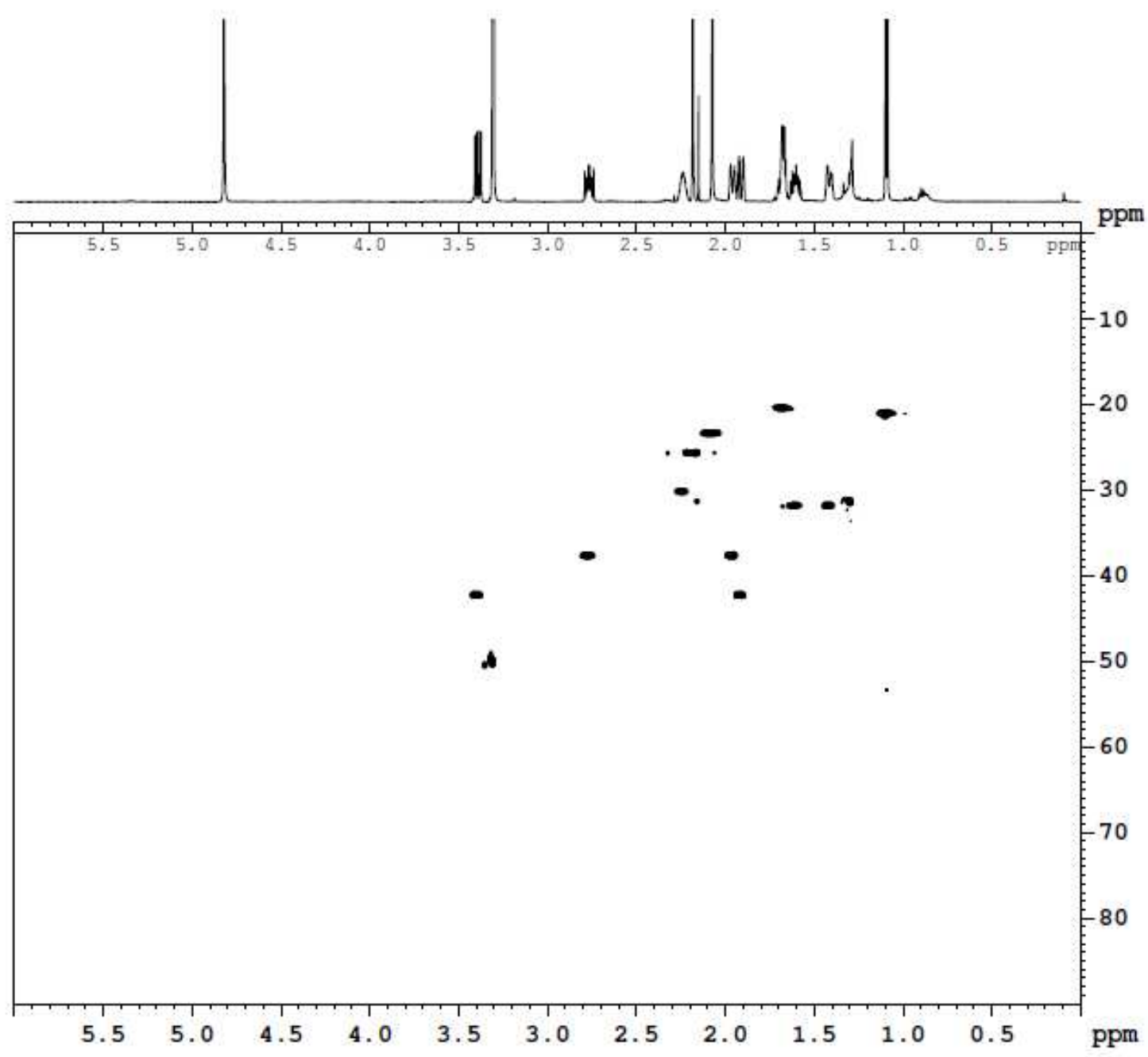


Compound $3 \mathrm{c}$

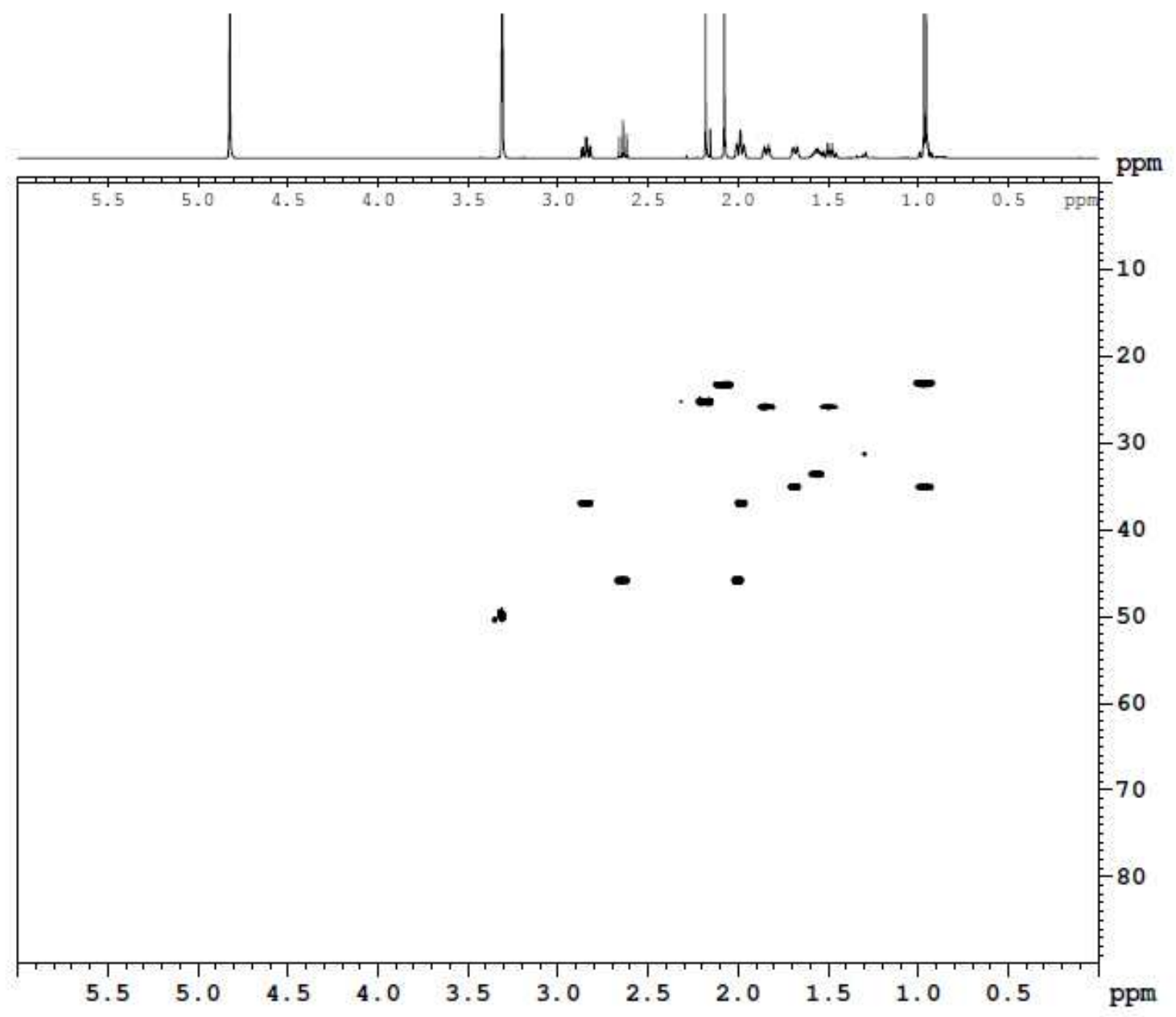


Cartesian Coordinates of GS- $\left(5 R^{*}, 6 S^{*}\right)-1-S_{a x}$ in chloroform

37

000000010.000000

N $0.5308-1.2866 \quad 0.1594$

$\mathrm{N}-0.8346-1.5264 \quad 0.1057$

$\mathrm{N}-2.8916-0.5014 \quad 0.0322$

O $2.6046-2.2358 \quad 0.2472$

O -3.38051 .72600 .0763$

S - $0.6344 \quad 1.1012 \quad 0.1160$

C $3.12532 .2306 \quad 0.3054$

C $2.24191 .9178 \quad 1.5202$

H 2.79502 .08132 .4524

C 1.73770 .46851 .4807

H $2.5867-0.2202 \quad 1.5458$

H 1.07720 .25962 .3294

C $0.9937 \quad 0.1400 \quad 0.1687$

C $1.8845 \quad 0.4629-1.0653$

H $2.7503-0.2005-0.9477$

C $2.39291 .9171-1.0059$

H $3.05712 .0899-1.8618$

H $1.54652 .6085-1.1352$

C $1.3801-2.3728 \quad 0.1958$

C $0.7230-3.73660 .1696$

H $0.0961-3.8612-0.7181$

H $0.0733-3.87861 .0386$

H $1.5145-4.48740 .1742$

C - $1.5135-0.4363 \quad 0.0818$

$\begin{array}{llll}C & -3.7722 & 0.5642 & 0.0354\end{array}$

C -5.2265 $0.1646-0.0143$

H -5.4877 -0.45840 .8486$

H -5.4392 $-0.4122-0.9213$

H -5.8429 $1.0636-0.0075$

$\mathrm{H}-3.2703-1.44730 .0063$

H 4.04271 .62800 .3602

H $3.4373 \quad 3.28190 .3222$

H 1.38822 .61011 .5429

C $1.23070 .1474-2.4135$

H $0.8625-0.8835-2.4568$

H $1.96380 .2670-3.2192$

H $0.38990 .8163-2.6285$

Cartesian Coordinates of GS- $\left(5 R^{*}, 6 S^{*}\right)-1-S_{\text {eq }}$ in chloroform 37

000000010.000000

N $0.5162-1.0990-0.0201$

N $-0.8068-1.38790 .3507$

$\mathrm{N}-2.9353-0.5083 \quad 0.3435$

O $2.4203-2.0758-0.8610$

O $-3.64581 .4713-0.5414$

S $-0.8511 \quad 1.0691-0.5771$

C $0.8238 \quad 0.3642-0.0125$

C $1.96710 .8498-0.9591$

H $1.92720 .2312-1.8614$

C $3.37890 .6848-0.3304$

H $3.6413-0.3740-0.3505$

H $4.0967 \quad 1.2045-0.9778$

C 3.49441 .19741 .1095

H 4.50160 .98731 .4899

H 3.36882 .28861 .1518

C 2.43490 .53011 .9906 
H 2.50130 .88743 .0252

H $2.6051-0.55522 .0203$

C 1.03370 .83371 .4482

H 0.26120 .38022 .0780

H 0.86671 .91661 .4789

C $1.76322 .3178-1.3925$

H $2.61252 .6320-2.0085$

H $1.70563 .0075-0.5438$

H $0.86222 .4546-1.9994$

C $1.2746-2.1806-0.4279$

C $0.6036-3.5362-0.3091$

H -0.2652 -3.6064 -0.9709

H $0.2454-3.7241 \quad 0.7067$

H $1.3409-4.2890-0.5928$

C -1.5858 -0.39490 .0883$

$\begin{array}{llll}C-3.9109 & 0.4284 & 0.0464\end{array}$

$\begin{array}{llll}\text { C }-5.2974 & 0.0417 & 0.4977\end{array}$

H -5.3135 $-0.1757 \quad 1.5712$

H -5.6349 $-0.8572-0.0308$

$\begin{array}{llll}H & -5.9836 & 0.8610 & 0.2825\end{array}$

H -3.2178 -1.3893 0.7718

Cartesian Coordinates of GS- $\left(5 R^{*}, 6 R^{*}\right)-1-S_{a x}$ in chloroform 37

000000010.000000

N $0.5133-1.2709-0.0949$

N $-0.8260-1.50330 .2149$

$\mathrm{N}-2.8977-0.4994 \quad 0.2754$

O $2.5143-2.2842-0.5607$

O $-3.46821 .5916-0.4349$

S - $-0.71551 .0302-0.4780$

C $0.93770 .1610-0.1465$

C 1.48560 .65581 .2299

H 0.65780 .57451 .9467

C $1.88482 .1481 \quad 1.1353$

H 0.98182 .75991 .0093

H 2.32742 .45222 .0916

C $2.85402 .4414-0.0188$

H $3.06653 .5165-0.0588$

H 3.81621 .94210 .1576

C $2.27371 .9677-1.3576$

H $2.99702 .1221-2.1668$

H $1.39362 .5756-1.6137$

C $1.89360 .4786-1.3118$

H $1.42240 .1699-2.2513$

H $2.7908-0.1328-1.1955$

C $2.6398-0.19831 .7836$

H $2.9730 \quad 0.22742 .7374$

H $3.5033-0.24101 .1150$

H $2.3276-1.22801 .9772$

C $1.3144-2.3774-0.3034$

C $0.6261-3.7232-0.1918$

H $0.1762-3.86110 .7959$

H $1.3800-4.4926-0.3667$

$\mathrm{H}-0.1804-3.8216-0.9247$

C -1.5385 -0.44660 .0448$

C -3.8114 0.50940 .0302

$\begin{array}{llll}C & -5.2367 & 0.1534 & 0.3741\end{array}$

H -5.5832 -0.6835 -0.2428

H $-5.8727 \quad 1.01960 .1905$

H -5.3238 -0.1465 1.4243 
H $-3.2399-1.39910 .6109$

Cartesian Coordinates of GS- $\left(5 R^{\star}, 6 R^{\star}\right)-1-S_{\text {eq }}$ in chloroform 37

000000010.000000

N $0.4985-0.9980-0.0332$

$\mathrm{N}-0.8363-1.3748-0.1691$

$\mathrm{N}-2.9964-0.5760-0.1822$

O $2.6311-1.8195 \quad 0.0557$

O -3.6964 $1.5641 \quad 0.1712$

S -0.9231 $1.2254 \quad 0.1397$

C $3.56371 .5598-0.6962$

C $2.78520 .7823-1.7633$

H $3.00621 .1775-2.7627$

C $1.27430 .9045-1.5172$

H $0.99061 .9575-1.6438$

H $0.70090 .3422-2.2633$

C $0.79760 .4766-0.1064$

C 1.70901 .06581 .0218

H 1.41962 .12801 .0516

C 3.22461 .05730 .7125

H 3.71531 .69231 .4621

H 3.60960 .04390 .8373

C $1.4183-2.02460 .0069$

C $0.8540-3.4310-0.0005$

H $0.3087-3.6344-0.9273$

H $0.1496-3.58610 .8217$

H $1.6935-4.12200 .0922$

C -1.6349 -0.3718 -0.0886

C -3.9761 $0.3912-0.0574$

C -5.3847 -0.1231 -0.2206

H -5.6156 -0.86620 .5510$

H -5.5133 - $0.6033-1.1969$

H $-6.08080 .7113-0.1336$

H -3.2769 -1.5434 -0.3380

H $3.32402 .6306-0.7797$

H $4.64311 .4702-0.8703$

H $3.0898-0.2682-1.7509$

C 1.42930 .47382 .4070

H 0.36710 .51432 .6682

H 1.97861 .03763 .1691

H $1.7606-0.56842 .4665$

Cartesian Coordinates of GS- $\left(5 R^{\star}, 6 R^{\star}\right)-\mathbf{2}-\mathrm{S}_{a x}$ in chloroform 46

000000010.000000

N $0.1635-1.39880 .3340$

N - $1.1929-1.5760 \quad 0.1074$

$\mathrm{N}-3.1970-0.4647-0.1011$

O $2.1644-2.4508 \quad 0.6416$

O $-3.6012 \quad 1.77790 .0088$

S -0.9231 1.02480 .4182

C 2.66861 .83991 .8679

C 1.49351 .33822 .7083

H 1.78031 .23003 .7611

C $0.9999-0.01052 .1811$

H $1.7832-0.76412 .3161$

H $0.1226-0.35032 .7431$

C $0.6356-0.01450 .6729$

C $1.84350 .5066-0.1820$ 


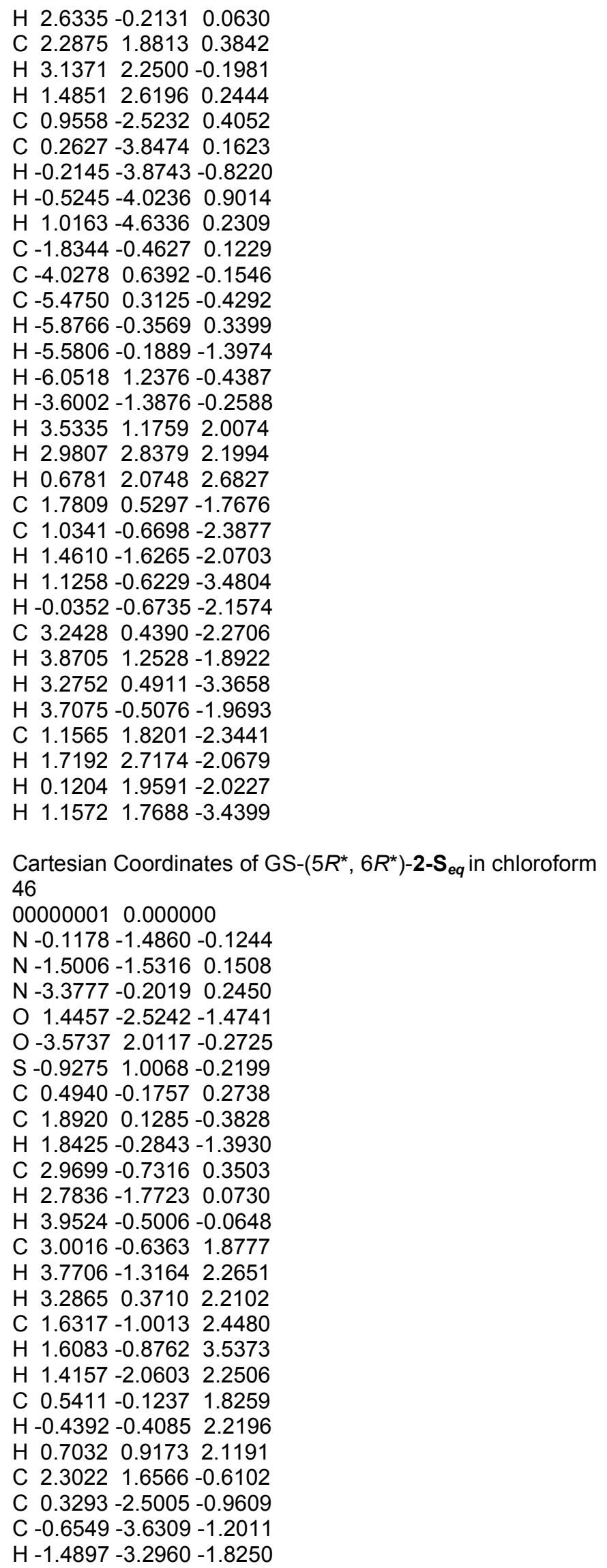

H $2.6335-0.21310 .0630$

C $2.2875 \quad 1.88130 .3842$

H $3.13712 .2500-0.1981$

H $1.48512 .6196 \quad 0.2444$

C $0.9558-2.52320 .4052$

$\mathrm{H}-5.8766-0.35690 .3399$

H -5.5806 -0.1889 -1.3974

H -6.0518 $1.2376-0.4387$

-3.6002 -1.3876 -0.2588

H $-0.0352-0.6735-2.1574$

C $3.24280 .4390-2.2706$

H $3.87051 .2528-1.8922$

$3.27520 .4911-3.3658$

H $3.7075-0.5076-1.9693$

C $1.15651 .8201-2.3441$

H $1.71922 .7174-2.0679$

H $0.12041 .9591-2.0227$

46

$\mathrm{N}-0.1178-1.4860-0.1244$

$\mathrm{N}-1.5006-1.53160 .1508$

$\mathrm{N}-3.3777-0.20190 .2450$

O $1.4457-2.5242-1.4741$

$\begin{array}{llll}1.45737 & 2.0117 & -0.2725\end{array}$

$\begin{array}{lll}-0.9275 & 1.0068 & -0.2199\end{array}$

$3.0016-0.6363-1.877$

H $3.7706-1.31642 .2651$

H 3.28650 .37102 .2102

C $1.6317-1.00132 .4480$

$1.6083-0.87623 .5373$

H $1.4157-2.06032 .2506$

C $0.5411-0.12371 .8259$

- $-0.4392-0.40852 .2196$

$\begin{array}{lll}0.7032 & 0.91732 .1191\end{array}$

$\mathrm{H}-1.4897-3.2960-1.8250$ 


\begin{abstract}
H - $1.0838-4.0105-0.2706$
H $-0.1128-4.4237-1.7196$

C -2.0184 $-0.3528 \quad 0.0760$

$\begin{array}{lll}\text { C }-4.1059 & 0.9646 & 0.0779\end{array}$

$\begin{array}{llll}\text { C }-5.5807 & 0.8182 & 0.3622\end{array}$

H -5.7448 $0.5517 \quad 1.4123$

$\mathrm{H}-6.02170 .0281-0.2560$

H -6.0803 1.76280 .1474

H -3.8792 -1.06450 .4543$

C $1.6272 \quad 2.1867-1.8993$

H $1.9307 \quad 1.5939-2.7710$

H $1.93153 .2240-2.0842$

H $0.53642 .1727-1.8621$

C $3.82361 .7504-0.8790$

H $4.14701 .0544-1.6621$

H 4.42461 .56200 .0158

H 4.06852 .7622 -1.2224

C 1.99682 .62450 .5539

H 2.36523 .62670 .3013

H $2.49742 .3257 \quad 1.4805$

H 0.92782 .72770 .7633
\end{abstract}

Cartesian Coordinates of GS- $\left(5 R^{*}, 6 S^{*}\right)-2-S_{a x}$ in chloroform 46

000000010.000000

N $0.1036-1.2574-0.4226$

$\mathrm{N}-1.1300-1.4414 \quad 0.2163$

$\mathrm{N}-3.1634-0.4223 \quad 0.5717$

O $1.8156-2.3732-1.4712$

O $-3.9413 \quad 1.5355-0.3059$

S $-1.2105 \quad 0.9705-0.8193$

C $0.5105 \quad 0.1605-0.5974$

C 1.15170 .82510 .6896

H $0.34450 .8597 \quad 1.4331$

C $1.44832 .3155 \quad 0.3427$

H 0.48492 .82640 .2241

H 1.92422 .80361 .1946

C $2.26432 .5530-0.9343$

H $2.3703 \quad 3.6323-1.0999$

H $3.28112 .1556-0.8271$

C $1.58281 .8994-2.1407$

H $2.20311 .9980-3.0394$

H $0.64072 .4223-2.3599$

C $1.31730 .4084-1.8865$

H $0.7793-0.0361-2.7300$

H $2.2658-0.1226-1.8127$

C $2.3107 \quad 0.06231 .4698$

C $0.7208-2.3950-0.9084$

C -0.0294 -3.6952 -0.6951

$\mathrm{H}-1.0034-3.6787-1.1939$

H $-0.2198-3.88150 .3661$

H $0.5829-4.4981-1.1091$

C -1.8861 -0.41090 .0536$

C - $4.1390 \quad 0.5424 \quad 0.3855$

C $-5.4404 \quad 0.2488 \quad 1.0902$

H -5.2795 0.08722 .1619

H -5.9010 -0.65750 .6801$

$\mathrm{H}-6.12001 .0890 \quad 0.9470$

H -3.4041 -1.2645 1.0934

C 2.88841 .00912 .5508

H 2.10181 .47813 .1549 
H 3.50991 .80482 .1287

H 3.52720 .43833 .2355

C $1.7162-1.13932 .2444$

H $0.8734-0.82942 .8747$

H $2.4776-1.56982 .9060$

H $1.3626-1.94241 .5962$

C $3.4933-0.44350 .6180$

H $4.2352-0.91451 .2767$

H 4.01190 .36840 .0965

H $3.1963-1.1915-0.1213$

Cartesian Coordinates of GS- $\left(5 R^{*}, 6 S^{*}\right)-2-S_{e q}$ in chloroform 46

000000010.000000

N $0.2037-1.13410 .4476$

$\mathrm{N}-1.1409-1.25270 .7906$

$\mathrm{N}-3.2559-0.4652 \quad 0.3301$

O $2.2679-2.0496 \quad 0.7947$

O $-3.85501 .1112-1.2027$

S - $1.13150 .5508-1.1082$

C $3.2546-0.0433-2.0667$

C $2.4174-1.3134-2.2123$

H $2.5226-1.7377-3.2193$

C $0.9397-0.9842-1.9834$

H $0.6085-0.3669-2.8280$

H $0.3190-1.8868-2.0151$

C $0.5584-0.2031-0.6841$

C $1.57550 .9683-0.3729$

H $1.30911 .7060-1.1458$

C $3.04540 .5702-0.6795$

H $3.66701 .4666-0.5870$

H $3.3929-0.14790 .0642$

C $1.0647-2.02261 .0550$

C $0.4547-2.95552 .0819$

$\mathrm{H}-0.3187-3.58721 .6358$

H $-0.0215-2.39832 .8942$

H $1.2580-3.57692 .4808$

C -1.8947 $-0.4744 \quad 0.0992$

C -4.1855 $0.3220-0.3223$

C -5.6074 $0.1227 \quad 0.1406$

H -5.7029 $0.3492 \quad 1.2081$

H -5.9229 -0.9157 -0.0108

H $-6.26210 .7836-0.4272$

H -3.5752 -1.1046 1.0568

H $2.97940 .6793-2.8501$

H $4.3194-0.2660-2.2100$

H 2.7600 -2.0656 -1.4974

C 1.49191 .76531 .0047

C $2.3707 \quad 1.13732 .1112$

H 2.11830 .09272 .3122

H 2.23761 .69573 .0453

H 3.43751 .17391 .8682

C 2.01833 .19730 .7399

H 1.37573 .73410 .0313

H 3.03783 .20640 .3404

H 2.03143 .77371 .6730

C 0.07011 .92911 .5809

H $-0.38170 .9765 \quad 1.8706$

H -0.6042 2.43640 .8864

H 0.12502 .54392 .4881 
Cartesian Coordinates of GS- $\left(5 R^{\star}, 7 R^{\star}\right)-3-S_{a x}$ in chloroform

37

000000010.000000

N $0.2281-1.45100 .0313$

$\mathrm{N}-1.1568-1.52330 .1008$

$\mathrm{N}-3.0732-0.24860 .1190$

O $2.1702-2.6448-0.0526$

O $-3.2648 \quad 2.0224 \quad 0.0310$

S $-0.6392 \quad 1.0547-0.0689$

C $0.8464-0.1046-0.1876$

C $1.8702 \quad 0.24190 .9111$

H 1.37300 .21841 .8876

C 2.55691 .60420 .6907

H 1.78782 .39090 .7533

C $3.18451 .6690-0.7128$

H $3.62372 .6609-0.8777$

H $4.01210 .9463-0.7691$

C $2.16061 .3587-1.8136$

H $2.64571 .3591-2.7966$

H $1.40082 .1522-1.8447$

C $1.4833-0.0005-1.5879$

H $0.7198-0.1872-2.3509$

H $2.2279-0.8011-1.6618$

C $0.9429-2.6295 \quad 0.0660$

C $0.1359-3.89510 .2620$

H $-0.4573-3.85301 .1804$

H $0.8362-4.73060 .3094$

$\mathrm{H}-0.5665-4.0471-0.5634$

C - $1.6990-0.3598 \quad 0.0616$

C - $\begin{array}{llll}\text {-3.8065 } & 0.9232 & 0.0941\end{array}$

$\begin{array}{llll}C & -5.3003 & 0.7230 & 0.1581\end{array}$

$\mathrm{H}-5.64210 .0562-0.6411$

H -5.79251 .69040 .0575$

H -5.5861 $0.2726 \quad 1.1156$

H -3.5710 - $1.1356 \quad 0.1856$

H $2.6248-0.55190 .9073$

C 3.58951 .87091 .7917

H 4.38361 .11401 .7776

H 3.12901 .85132 .7866

H 4.06172 .85191 .6634

Cartesian Coordinates of GS- $\left(5 R^{\star}, 7 R^{\star}\right)-3-S_{\text {eq }}$ in chloroform 37

000000010.000000

N $0.4641-0.9597-0.0869$

N - $-0.8124-1.24590 .4212$

$\mathrm{N}-2.9757-0.45320 .4626$

O $2.3650-1.9240-0.9271$

O $-3.8332 \quad 1.3444-0.6484$

S $-1.0370 \quad 1.0557-0.8217$

C 3.37881 .79150 .2025

C $3.15640 .9794-1.0801$

H $3.69131 .4496-1.9149$

C $1.66880 .8805-1.4653$

H $1.34631 .8769-1.7979$

H $1.5290 \quad 0.2075-2.3153$

C $0.7046 \quad 0.4913-0.3229$

C 1.05951 .22750 .9945

H 0.74012 .26840 .8654

C $2.55431 .2477 \quad 1.3864$ 


\begin{abstract}
H 2.62941 .97522 .2068
C $1.2484-2.0431-0.4232$

C $0.6604-3.4071-0.1200$

H $-0.2628-3.5773-0.6824$

H $0.4100-3.50990 .9398$

H $1.4049-4.1539-0.4008$

C - $1.6558-0.32810 .0896$

$\begin{array}{llll}C & -4.0117 & 0.3930 & 0.1042\end{array}$

C - $-5.34350 .0361 \quad 0.7156$

H -5.2903 $0.0811 \quad 1.8093$

H -5.6400 -0.98150 .4370$

H -6.0981 0.73890 .3620

H -3.1856 - $1.2707 \quad 1.0344$

H $3.09652 .8398 \quad 0.0273$

H 4.44621 .79730 .4580

H $3.5603-0.0273-0.9677$

H $0.4494 \quad 0.8152 \quad 1.8065$

C $3.0912-0.08491 .9293$

H 4.10710 .04702 .3218

H $3.1356-0.86811 .1668$

H $2.4687-0.45302 .7543$
\end{abstract}

Cartesian Coordinates of GS- $\left(5 R^{*}, 7 S^{*}\right)-3-S_{a x}$ in chloroform 37

000000010.000000

N $0.3440-1.4745-0.1317$

$\mathrm{N}-1.0385-1.5865-0.0575$

$\mathrm{N}-2.9865-0.3677 \quad 0.0538$

O $2.3110-2.6376-0.1224$

O $-3.25391 .8958-0.0402$

S -0.5819 $1.0051-0.0557$

C $3.37101 .5648 \quad 0.5697$

C 2.32281 .32911 .6667

H 2.80621 .31712 .6505

C $1.5857-0.00051 .4579$

H $2.2968-0.83051 .5415$

H $0.8173-0.14692 .2252$

C $0.9396-0.1094 \quad 0.0620$

C $1.9528 \quad 0.2286-1.0537$

H $2.6580-0.6070-1.0784$

C $2.75911 .5353-0.8471$

H $3.58731 .4838-1.5668$

C $1.0789-2.6427-0.1673$

C $0.2862-3.9282-0.2731$

H -0.3832 -3.9191 -1.1383

H -0.3408 $-4.0778 \quad 0.6115$

H $0.9990-4.7496-0.3618$

C - $1.6098-0.4373-0.0144$

C - $-3.7574 \quad 0.78010 .0418$

$\begin{array}{llll}C & -5.2425 & 0.5296 & 0.1344\end{array}$

H -5.4885 -0.01881 .0505$

H -5.5873 $-0.0688-0.7165$

H -5.7651 1.48630 .1352

$\begin{array}{lll}\mathrm{H}-3.4566 & -1.2712 & 0.0944\end{array}$

H $4.1400 \quad 0.78310 .6424$

H 3.88172 .52210 .7335

H 1.60112 .15681 .6938

H $1.4350 \quad 0.2447-2.0194$

C $1.98652 .8248-1.1722$

H $2.66773 .6842-1.1649$

H $1.18873 .0434-0.4543$ 
H $1.52632 .7764-2.1659$

Cartesian Coordinates of GS-(5R* $\left.7 S^{*}\right)-3-S_{e q}$ in chloroform 37

000000010.000000

N $0.2724-1.1112-0.0856$

$\mathrm{N}-1.0863-1.3900 \quad 0.0696$

$\mathrm{N}-3.1756-0.4133 \quad 0.0817$

O $2.3315-2.0874-0.2928$

O -3.6855 $1.7614-0.3675$

S $-0.9599 \quad 1.2055-0.2699$

C 0.66860 .32340 .1074

C $1.71250 .8925-0.8872$

H $1.53480 .4579-1.8771$

C $3.20700 .7863-0.5063$

C $3.43641 .1440 \quad 0.9713$

H 4.48960 .97101 .2280

H 3.25342 .21961 .1260

C $2.52280 .3379 \quad 1.9023$

H 2.70690 .61192 .9484

C 1.04900 .61821 .5759

H 0.37460 .06002 .2353

H 0.85101 .68251 .7576

C $1.1067-2.1975-0.2433$

C $0.4274-3.5467-0.3635$

H -0.2969 -3.5610 -1.1831

$\mathrm{H}-0.1228-3.7953 \quad 0.5491$

H $1.2049-4.2916-0.5404$

C -1.8031 -0.3259-0.0184

C -4.0673 $0.6313-0.0789$

$\begin{array}{llll}C-5.5128 & 0.2573 & 0.1335\end{array}$

H -5.6751 -0.07941 .1636$

H -5.8076 -0.5583 -0.5360

H $-6.13811 .1284-0.0627$

H -3.5381 -1.34700 .2709$

H $1.48141 .9631-0.9842$

H $2.7419-0.7307 \quad 1.8027$

H $3.5105-0.2534-0.6439$

C $4.04451 .6700-1.4383$

H $5.11301 .5778-1.2099$

H $3.77652 .7306-1.3407$

H $3.90881 .3897-2.4903$

Cartesian Coordinates of GS-sCT $\mathbf{C T}_{0}-\mathrm{S}_{a x}$ in chloroform 34

000000010.000000

N $0.6153-1.28190 .0566$

$\mathrm{N}-0.7476-1.5312-0.0454$

S - $-0.5696 \quad 1.08310 .2257$

C $3.15252 .2684-0.1677$

C 2.48791 .93401 .1749

H 3.19182 .09322 .0001

C 1.99040 .48021 .2185

H 1.46890 .27462 .1596

C $1.0598 \quad 0.14680 .0366$

C $1.7224 \quad 0.5072-1.3088$

C $2.21551 .9621-1.3447$

H $2.72462 .1449-2.2983$

H $1.35472 .6451-1.3179$

C - $1.4371-0.4494 \quad 0.0232$ 
$\begin{array}{lrrr}\text { H } & 4.0736 & 1.6776 & -0.2744 \\ \text { H } & 3.4520 & 3.3230 & -0.1883 \\ \text { H } & 1.6478 & 2.6214 & 1.3536 \\ \text { H } & 1.0182 & 0.3136 & -2.1256 \\ \text { H } & 2.8417 & -0.2044 & 1.1569 \\ \text { H } & 2.5721 & -0.1732 & -1.4395 \\ \text { C } & 1.4769 & -2.3584 & 0.0142 \\ \text { O } & 2.7004 & -2.2074 & -0.0046 \\ \text { C } & 0.8332 & -3.7285 & 0.0033 \\ \text { H } & 0.2366 & -3.8773 & -0.9023 \\ \text { H } & 0.1574 & -3.8602 & 0.8534 \\ \text { H } & 1.6320 & -4.4708 & 0.0430 \\ \text { H } & -3.1868 & -1.4521 & -0.2055 \\ \text { N } & -2.8127 & -0.5144 & -0.0647 \\ \text { C } & -3.6967 & 0.5466 & 0.0103 \\ \text { C } & -5.1447 & 0.1602 & -0.1641 \\ \text { H } & -5.4554 & -0.5405 & 0.6191 \\ \text { H } & -5.3026 & -0.3284 & -1.1320 \\ \text { H } & -5.7612 & 1.0576 & -0.1077 \\ \text { O } & -3.3126 & 1.6955 & 0.2041\end{array}$

Cartesian Coordinates of GS-sCT $\mathbf{C T}_{\mathbf{0}}-\mathrm{S}_{e q}$ in chloroform 34

000000010.000000

N $0.5908-0.9732-0.0907$

N $-0.7261-1.3440 \quad 0.1805$

N - $2.8905-0.55330 .2252$

O $2.7178-1.7835-0.3086$

O $-3.62881 .5057-0.4132$

S $-0.85341 .1985-0.4406$

C $0.86960 .5025-0.1005$

C $1.7841 \quad 1.0198-1.2424$

H $1.57780 .4436-2.1502$

C $3.29751 .0784-0.9567$

H $3.71890 .0760-1.0331$

H $3.76281 .6956-1.7358$

C 3.61151 .65450 .4302

H 4.69121 .60300 .6167

H 3.33952 .72050 .4652

C 2.84390 .90411 .5260

H 3.07011 .32952 .5116

H $3.1565-0.14551 .5438$

C $1.3298 \quad 1.0081 \quad 1.2874$

H 0.76360 .47652 .0609

H 1.03902 .06401 .3631

C $1.5143-1.9943-0.1631$

C $0.9620-3.4017-0.0626$

H $0.1964-3.5891-0.8210$

H $0.4920-3.57330 .9108$

H $1.7962-4.0924-0.1959$

C - $1.5396-0.36010 .0269$

$\begin{array}{llll}\text { C }-3.8846 & 0.3836 & 0.0127\end{array}$

C -5.2743 -0.09330 .3541$

H -5.3642 $-0.2643 \quad 1.4330$

$\mathrm{H}-5.5027-1.0359-0.1550$

$\begin{array}{llll}\mathrm{H} & -5.9959 & 0.6657 & 0.0515\end{array}$

H -3.1526 -1.4887 0.5337

H $1.44972 .0448-1.4525$

Cartesian Coordinates of GS-sCT $\mathbf{T}_{1} \mathrm{~S}_{a x}$ in chloroform 27 
000000010.000000

$\begin{array}{llll}N & 1.2543 & 0.0409 & 0.0000\end{array}$

N 1.98221 .21970 .0000

S -0.5197 2.00010 .0000

C -2.9862 -1.05690 .0000$

C $-2.3929-0.4251-1.2673$

H - $2.7736-0.9305-2.1623$

C - $0.8570-0.4917-1.2701$

H -0.4451 $0.0070-2.1539$

C - $-0.2478 \quad 0.1310 \quad 0.0000$

C $-0.8570-0.49171 .2701$

C -2.3929 -0.42511 .2673$

H -2.7736 $-0.9305 \quad 2.1623$

H -2.7176 $0.6226 \quad 1.3445$

C 1.22262 .24750 .0000

$\mathrm{H}-2.7781-2.13610 .0000$

H $-4.0774-0.94800 .0000$

H -2.7176 $0.6226-1.3445$

H - 0.44510 .00702 .1539

H - $0.5325-1.5375-1.2976$

H -0.5325 -1.5375 1.2976

H $1.64003 .2482-0.0000$

C $1.9501-1.15610 .0000$

O $1.3642-2.23780 .0000$

C $3.4602-1.05260 .0000$

H $3.8218-0.50950 .8782$

H $3.8218-0.5095-0.8782$

H $3.8604-2.06750 .0000$

Cartesian Coordinates of GS-sCT $\mathbf{C T}_{1}-\mathrm{S}_{\text {eq }}$ in chloroform 27

000000010.000000

N $1.0014-0.39190 .0000$

N $2.3124 \quad 0.0616 \quad 0.0000$

S 0.88652 .24150 .0000

C $-0.1134 \quad 0.6384 \quad 0.0000$

C - $-0.9776 \quad 0.6322 \quad 1.2896$

$\mathrm{H}-0.3310 \quad 0.4022 \quad 2.1436$

C $-2.2206-0.2745 \quad 1.2657$

$\mathrm{H}-1.9223-1.32371 .3223$

$\mathrm{H}-2.8189-0.05312 .1588$

C $-3.0565-0.04770 .0000$

$\mathrm{H}-3.9252-0.7170 \quad 0.0000$

$\mathrm{H}-3.45360 .97890 .0000$

C - $-2.2206-0.2745-1.2657$

$\mathrm{H}-2.8189-0.0531-2.1588$

$\mathrm{H}-1.9223-1.3237-1.3223$

C - $0.97760 .6322-1.2896$

$\mathrm{H}-0.3310 \quad 0.4022-2.1436$

$\mathrm{H}-1.3191 \quad 1.6646-1.4381$

C 2.38511 .33740 .0000

$\mathrm{H}-1.3191 \quad 1.6646 \quad 1.4381$

H 3.34521 .84140 .0000

C $0.8312-1.76630 .0000$

O $-0.2825-2.2841 \quad 0.0000$

C $2.0955-2.5996 \quad 0.0000$

H $2.7135-2.3890 \quad 0.8777$

H $2.7135-2.3890-0.8777$

H $1.7951-3.64850 .0000$

Cartesian Coordinates of GS-sCT $\mathbf{C T}_{2}-\mathrm{S}_{a x}$ in chloroform 


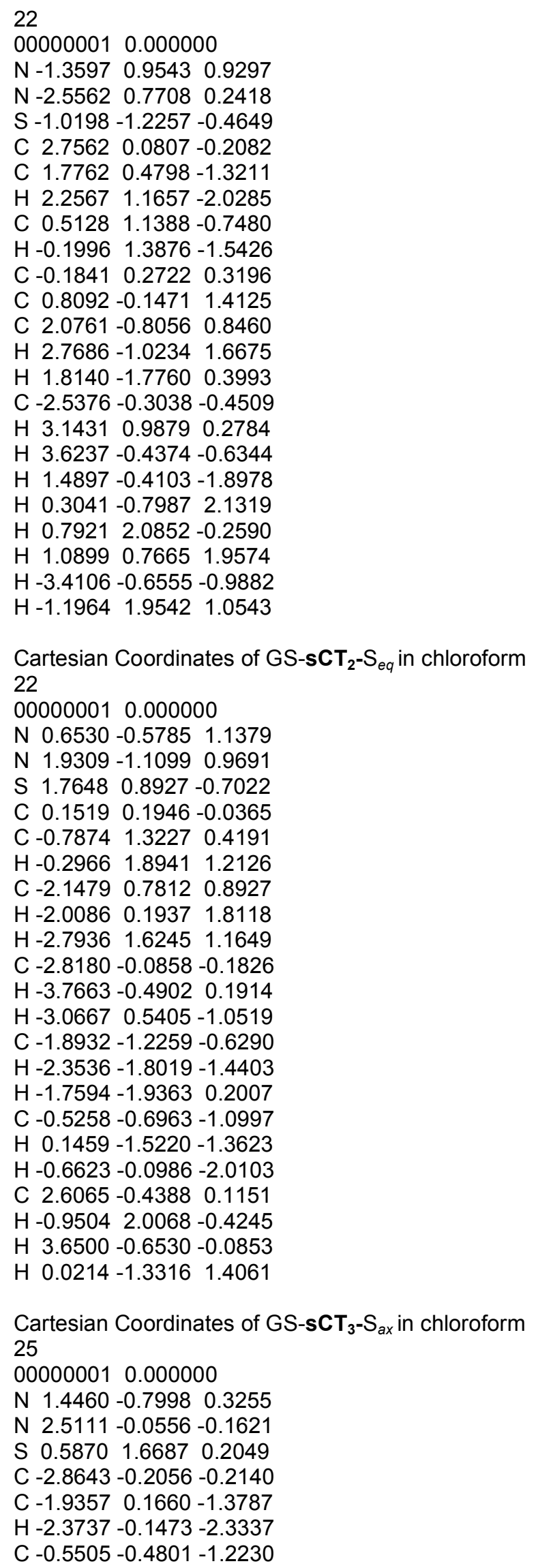

Cartesian Coordinates of GS-s $\mathbf{C} \mathbf{T}_{3}-\mathrm{S}_{a x}$ in chloroform 25 


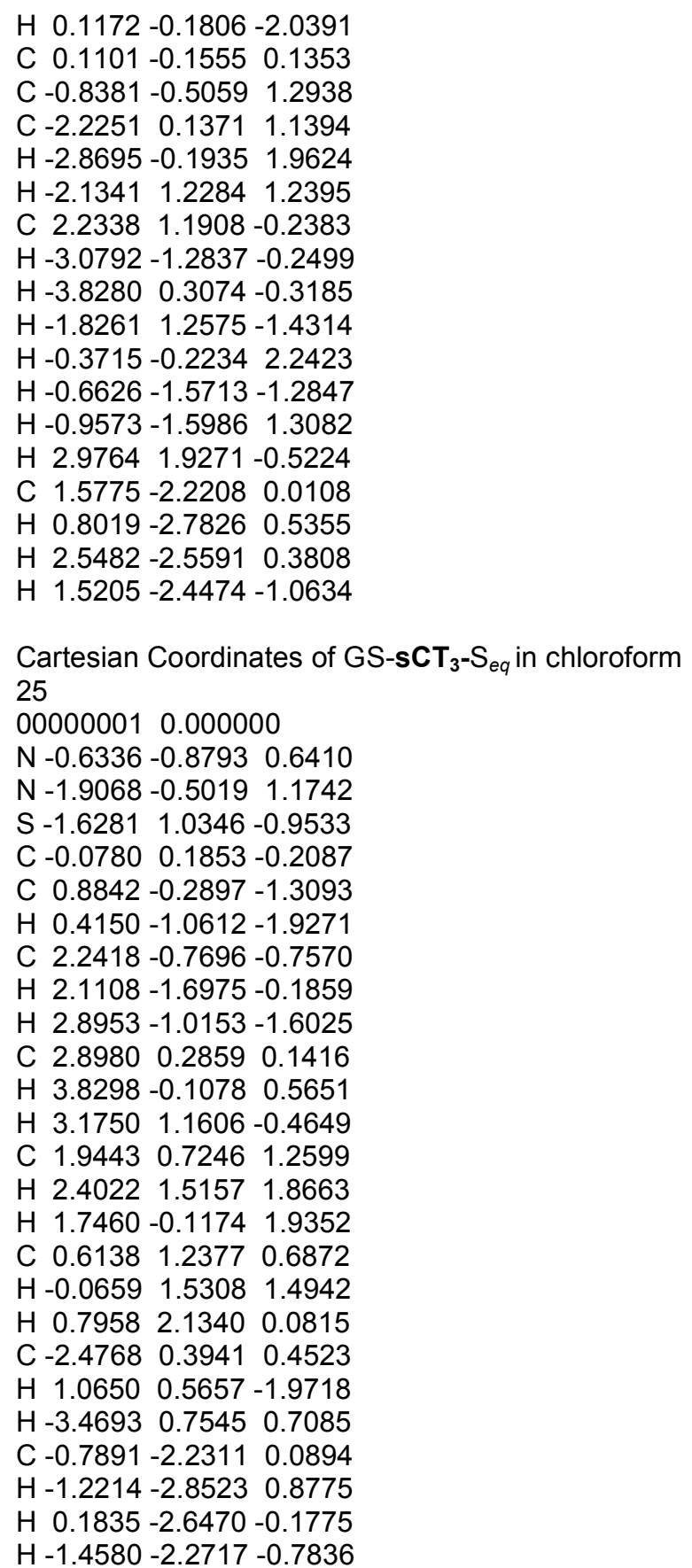

